NASA Contractor Report 4075

\title{
Structural Concepts for Large Solar Concentrators
}

John M. Hedgepeth and Richard K. Miller

CONTRACT NAS1-17536

JUNE 1987 
NASA Contractor Report 4075

\section{Structural Concepts for Large Solar Concentrators}

John M. Hedgepeth and Richard K. Miller Astro Aerospace Corporation

Carpinteria, California

Prepared for

Langley Research Center

under Contract NAS1-17536

\section{Nush}

National Aeronautics

and Space Administration

Sclentific and Technical Information Office 


\section{FOREWORD}

Astro Aerospace Corporation is conducting studies on design concepts for large space structures. This is one of a series of reports issued as part of those studies under Contract No. NAS1-17536. 


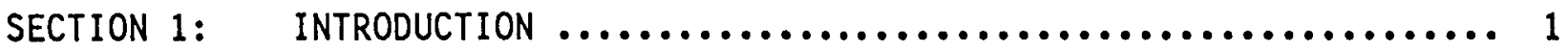

SECTION 2: SOLAR CONCENTRATOR CONCEPTS $\ldots \ldots \ldots \ldots \ldots \ldots \ldots \ldots \ldots \ldots, 4$

Collecting Surface $\ldots \ldots \ldots \ldots \ldots \ldots \ldots \ldots \ldots \ldots \ldots \ldots \ldots \ldots, \ldots, 4$

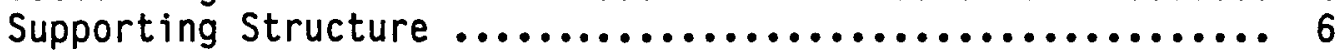

Mounting Structure $\ldots \ldots \ldots \ldots \ldots \ldots \ldots \ldots \ldots \ldots \ldots \ldots \ldots \ldots \ldots, 9$

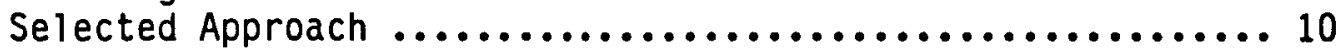

SECTION 3: EFFECTS OF GEOMETRIC ERRORS ON COLLECTOR EFFICIENCY ....... 11

Effects of Random Slope Errors on the Effective Concentration Ratio of a Continuous-Paraboloidal

Reflector ..................................... 11

Effects of Location, Orientation and Size of Nominally

Square Spherical Panels on the Effective Concentration

Ratio................................................ 15

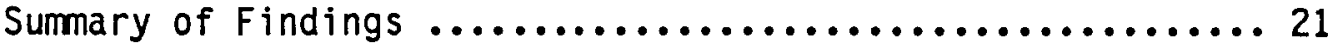

SECTION 4: EXAMPLE OF TRUSS-SUPPORTED CONCEPTUAL DESIGN $\ldots \ldots \ldots \ldots \ldots \ldots 22$

SECTION 5: CONCLUDING REMARKS $\ldots \ldots \ldots \ldots \ldots \ldots \ldots \ldots \ldots \ldots \ldots \ldots \ldots \ldots . \ldots \ldots$

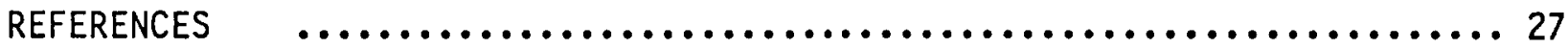

APPENDIX $\quad$............................................ 50 


\section{LIST OF FIGURES}

Figure 1a. Symetric antenna reflector configuration (QUASAT - 20-m

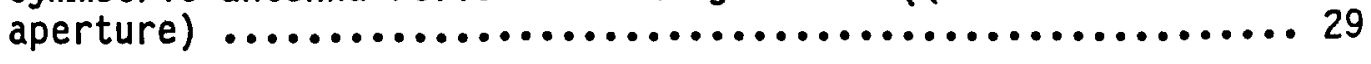

Figure 1b. Offset-fed antenna reflector configuration $\ldots . . . . . . . . . .29$

Figure 2. Main panel double ring configuration $\ldots \ldots \ldots \ldots \ldots \ldots \ldots . . . . . .30$

Figure 3. Qualitative comparative unit costs for panel-type solar

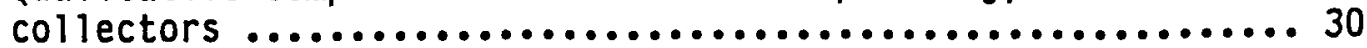

Figure 4. Mosaic reflector of spherical segments assembled by RMS from Shuttle .................................. 31

Figure 5. Assembly of integrated panel-truss modules $\ldots \ldots \ldots \ldots \ldots \ldots . . . .31$

Figure 6. Sequential deployment of connected panel-truss modules $\ldots . . .32$

Figure $7 . \quad$ LDR deployment sequence $\ldots \ldots \ldots \ldots \ldots \ldots \ldots \ldots \ldots \ldots \ldots \ldots \ldots$

Figure 8. Synchronously deployable concept (CREST) for stiff-panel

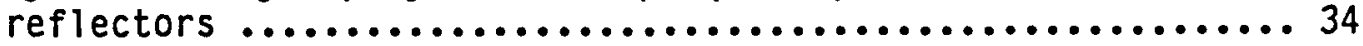

Figure 9. Assembly of the LPR infrared telescope $\ldots \ldots \ldots \ldots \ldots \ldots \ldots . \ldots 35$

Figure 10. Deployment of preintegrated support-truss modules for the

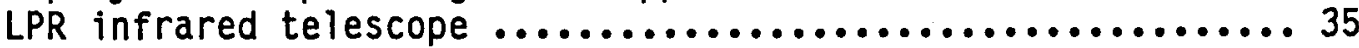

Figure 11. Support-truss for a seven-tile module of the LPR infrared

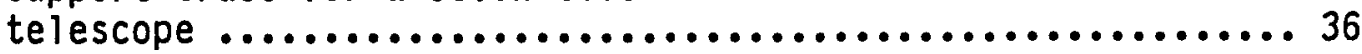

Figure 12. Application of Pactruss to the support structure for the primary mirror of the JPL low-cost LDR .................... 37

Figure 13. Synchronously deployable Pactruss concept $\ldots \ldots \ldots \ldots \ldots \ldots \ldots 38$

Figure 14. Deployment of conceptual model of triangular cell .......... 39

Figure 15. Views of Pactruss for offset paraboloid ................ 40

Figure 16. Geometry of a continuous paraboloid reflector $\ldots . . . . . . . . .41$

Figure 17. Intersection of the sun's image and the receiver disk in the receiver plane 
Figure 18. Mean effective concentration ratio vs. geometric concentration ratio $C$ with rms surface slope error

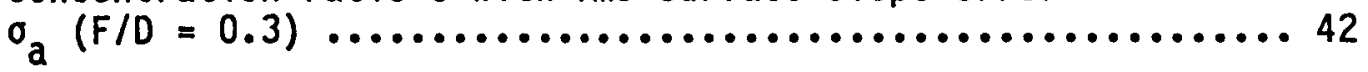

Figure 19. Mean effective concentration ratio vs. geometric concentration ratio $C$ with rms surface slope error

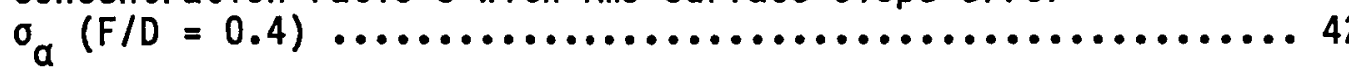

Figure 20. Mean effective concentration ratio vs. geometric concentration ratio $C$ with mis surface slope error $\sigma_{a}(F / D=0.5)$

Figure 21. Mean effective concentration ratio vs. geometric concentration ratio $C$ with rms surface slope error $\sigma_{\alpha}(F / D=0.6)$

Figure 22. Mean effective concentration ratio vs. geometric concentration ratio $\mathrm{C}$ with rms surface slope error

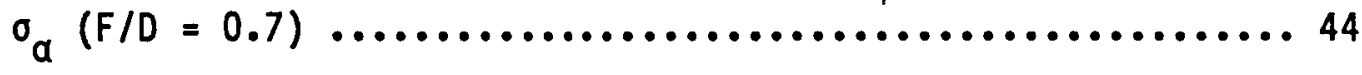

Figure 23. Effect of $F / D$ on required geometric concentration ratio $C$ with rms surface slope error $\sigma_{\alpha}$ $\left(E\left[C_{\text {eff }}\right]=1,000\right)$

Figure 24. Effect of $F / D$ on required geometric concentration ratio $C$ with rms surface slope error $\sigma_{\alpha}$ $\left(E\left[C_{\text {eff }}\right]=1,500\right)$

Figure 25. Effect of $F / D$ on required geometric concentration ratio $C$ with rms surface slope error $\sigma_{\alpha}$ $\left(\mathrm{E}\left[\mathrm{C}_{\text {eff }}\right]=1,000\right)$

Figure 26. Nominally square shallow spherical panel with side length "a"

Figure 27. Location and orientation geometry of square spherical panels 46

Figure 28. Effect of panel orientation angle $\psi$ on maximum panel size $a_{m} / F$ vs. panel location angle $\theta_{c}$

Figure 29. Effects of pivoting the panel so that $p$ and $c$ are not coincident, on the maximum allowable panel size for the case where $c_{\text {eff }}=2,000, p_{p}=F$, and $\psi=45$ degreesf 


\section{LIST OF FIGURES, concluded}

Figure 30. Maximum panel size $a_{p} / F$ vs. panel location angle $\theta_{c}$ with different panel distances

Figure 31. Maximum panel size $a_{m} / F$ vs. panel location angle $\theta_{c}$ with different panel distances $\rho_{p} / F \ldots \ldots \ldots \ldots \ldots \ldots \ldots \ldots \ldots \ldots 48$

Figure 32. Identical-panel concept perspective $\ldots \ldots \ldots \ldots \ldots \ldots \ldots \ldots . . . .49$

Figure 33. Pactruss-supported, identical-panel concept $\ldots \ldots \ldots \ldots \ldots \ldots . \ldots 9$ 
SECTION 1

INTRODUCTION

Meeting the power requirements of future space missions such as the Space Station will require advances in space power technology. Most currently operating satellites have relatively low power requirements and use a low efficiency photovoltaic solar-array system to supply all of the power needs. Batteries are used during the part of the orbit in the earth's shadow. However, preliminary estimates of the power requirements for the proposed Space Station indicate that a photovoltaic power system would require an area of solar cells of thousands of square meters. Such arrays would have a large aerodynamic drag and would consequently affect the station operation and the selection of the station architecture. Station operation could be greatly enhanced and constraints on station architecture minimized by reducing the need for such large areas. Preliminary results from recent studies have shown the potential for significant area reductions using a solar thermal approach. In this approach, concentrated solar energy is used to heat a fluid which then drives a power-producing heat engine.

The potential reduction in area using a solar thermal approach comes from two sources. One is the higher conversion efficiency of a solar thermal system. This advantage has been known for years and is controlled by fundamental physical laws. The second is in the overall power system operation and design. Recent studies have shown that the overall system operation would benefit by storing energy (for use while in the earth's shadow) as thermal energy rather than as electrical energy. The increase in efficiency due to the combination of storage and conversion can reduce the surface area required for the Space Station power system by a factor of five.

The advantage of the photovoltaic system is its simplicity and insensitivity to geometric errors in orientation of the solar array. In addition, a large amount of development work has resulted from the frequent use of the technique. The structural needs and approaches have been studied 
in detail and knowledge is well advanced on the weight, cost and performance that can be expected.

In the solar-thermal approach, the solar radiation must be concentrated at a receiver so that high temperatures are produced. The amount of concentration required is dependent on the type of power conversion chosen, but ranges from a value of 1,000 to 2,000. Such high concentration ratios place much more stringent demands on the concentrator surface and its supporting structure than exist for the photovoltaic structural components. Consequently, the performance of a solar-thermal power system is very dependent on the performance and weight of the concentrator structure.

During the 1960s, a large number of investigations were conducted on the solar concentrator and its support structure. This early work is reported in a variety of forms. Examples can be found in the Power systems Conference Proceedings (References 1 and 2). Various types of reflectors are discussed in the next section. The type that received the most advanced development consists of honeycomb-core-stiffened reflector panels. A deployable collector with such panels as petals reached the stage of prototype hardware (Reference 3).

The success of photovoltaic systems for low power requirements, the lack of space missions requiring large power levels, and budgetary constraints led to the cessation of work on solar-dynamic (as well as nuclear-dynamic) power systems in the late 1960s. Not until the middle 1970 s did interest rekindle, this time for terrestrial use, because of the disruptions in oil supply and price. In order to provide an alternative energy source, a multi-year program was undertaken, aimed at the construction of long-life, cost-effective paraboloidal-collector power-generation units. Several collector concepts have been developed and tested; the most successful consist of inexpensive, stiff reflector panels mounted on a truss supporting structure. (See Reference 4.) The current Space Station studies are benefitting from the technology developed for terrestrial application. (See Reference 5.)

In the present paper, various types of solar concentrators are discussed from a structural point of view. The discussion includes reflecting surfaces 
of a membrane type as well as stiffened panels. The methods for supporting the reflecting surface are described. The deep truss supporting structure is identified as providing high accuracy at reasonable cost, although on-orbit assembly is required to mount the reflector panels to the truss.

Ray-tracing analyses are performed to evaluate the tolerable amount of slope inaccuracy of the nominally paraboloidal reflecting surface of a concentrator. For the high concentration ratios (approximately 2,000) required for a Brayton-cycle heat engine, an rms slope error of three milliradians is acceptable. Next, a special nonparaboloidal reflector concept, composed of identical square, spherically curved panels efficiently located, is analyzed by ray tracing. Such a configuration should reduce the costs of panel fabrication.

Because the identical-panel concept with large panel sizes is shown to be feasible, a configuration is generated which combines the square panels with a Pactruss supporting structure. The resulting design is described and its mass breakdown is estimated. 
SECTION 2

SOLAR CONCENTRATOR CONCEPTS

In this section, attention is devoted to those concentrator configurations which are so large that they must be carried into orbit in a packaged condition. The collecting area is greater than about 100 square meters. Therefore, fixed dishes, which can be assembled and aligned on the ground prior to launch, are precluded.

The concentrator must not only be packaged, but also be compactly stowed for launch. The Space Station will require numerous Shuttle launches; the concentrator must not add unnecessarily to the number.

In general, a solar concentrator can be considered to be composed of three components: the collecting surface, the supporting structure, and the mounts which connect the concentrator to the rest of the spacecraft. It is convenient to consider each component separately.

\section{Collecting Surface}

Lenses - The surface can consist of a lens or a reflector. Lenses can be constructed of thin layers by using the fresnel principle. Indeed, the lens can be structurally a flat membrane and supported as a drumhead. The lenses have the advantage of being relatively insensitive to geometric errors. On the other hand, the energy must be transmitted through the lens material. Maintaining low loss throughout the long-term exposure to the space environment places stringent requirements on the lens material. In addition, the supporting structure will block the energy unless great care is taken.

Reflectors - The reflecting surface has been adopted almost universally for solar concentrator for terrestrial and space applications. The surface must be a highly specular reflector and must be oriented accurately in order to direct the reflected rays into the thermal receiver. Analyses such as those described in Section 3 show that the orientation accuracy required is 
not as great as that for many of the high-performance RF missions presently being undertaken. The sun subtends about nine milliradians in arc. Little is gained by demanding reflected-ray accuracies better than about three milliradians, even for a concentration ratio of 2,000 .

The achievement of highly specular, low-loss reflection for long-term space missions is a more difficult objective. Even with solid surfaces, about ten percent of the incident energy is lost; with metallized films the specular reflectivity is reduced to perhaps 80 percent after long exposure. The reflection losses are minimized in most collector arrangements by avoiding multiple reflections.

Film Surfaces - Film-type reflector surfaces are attractive from packing and weight standpoints. Furthermore, only the film surface affords the possibility of uncomplicated deployment without requiring on-orbit assembly. However, the surface must be doubly curved with positive Gaussian curvature. In order to achieve this, some method must be found to apply a pressure across the membrane. Inflation will accomplish this objective, but leakage will require prohibitive amounts of make-up inflatant unless the collector is very large (kilometer scale), the pressure is low, or the pressurization time is sma1l. The last alternative is probably the most feasible. Inflation is used to stretch the wrinkles from the packaged reflector. The resulting reflector is then stiff enough to maintain its shape, either because the surface material is a multilayered film or because it is a composite which is cured on orbit after inflation.

In any event, the film will operate at very low stress levels. Thus, the shape accuracy must be attained primarily by enforcing the correct geometry during fabrication. As is shown in the next section, efficient solar concentrators tend to be deep dishes; for these the in-surface stiffness dominates the structural behavior.

An alternate method of applying the shaping pressure is by electrostatic attraction. This technique avoids the gas leakage problem. It furthermore offers the opportunity of controlling the loading and hence, the shape. The latter controllability can be useful for shallow dishes, but not for the types 
needed for solar concentrators, unless the surface is divided into numerous panels.

Panel Surfaces - The alternative to the membrane reflector surface is an assemblage of stiff panels that are individually small enough to fit in the launch vehicle. Each panel is considered to be stiff enough to maintain its own shape.

There are two primary textures for the panel construction. One is the honeycomb sandwich; the other is the thin shell, stiffened or monocoque. If the shell is monocoque, the panel boundary must be dimensionaliy stable in order to preclude warping.

Panels can be constructed from a wide range of materials. Long-life stability of the reflective and dimensional properties would appear to favor metallic construction. The Sunflower petals (Reference 3) were essentially of aluminum construction, face sheets and honeycomb core. Recent work has dealt with graphite- and Kevlar-reinforced composites for the face sheets. Producing a smooth reflecting surface will probably require a shaping mandrel in either case.

Masses - The film surface has a unit mass of the order of $0.1 \mathrm{~kg} / \mathrm{m}^{2}$, whereas the panel concept will weigh at least one $\mathrm{kg} / \mathrm{m}^{2}$. If very $\mathrm{precise}$ surfaces are required, the mass can increase by another order of magnitude.

\section{Supporting Structure}

Film Surfaces - Concentrator configurations utilizing film reflector surfaces usually consist of a transparent pressure containment surface joined to the reflector at the rim. The containment surface can be shaped symmetrically to the reflector so that the pressurized vessel is lenticular. [See Figure 1(a) and (b) taken from Reference 6.] The rim must be capable of carrying compression without local crippling. It must also be stiff and stable enough to maintain the shape of the edge after the internal pressure is removed. Rims for inflated reflectors tend also to be inflated. Deployment is achieved by pressurizing in a controlled fashion. 
If electrostatic forces are used to tension the membrane, no frontal membrane is needed. On the other hand, a substantial back-up truss is required to support the electrostatic devices. In addition, the membrane and the charged devices must be shielded from the space plasma in order to avold electrical leakage.

Panels - The supporting structure for panels can be made up in a variety of ways. At one extreme, the panels are stiff enough to serve as their own structure. At the other, a separate back-up structure (a truss, for example) is furnished and the panels are attached to it. An intermediate configuration could be one in which the panels derive their main support from their own stiffness but are externally supported at several points, say at the rim, in order to remove large-scale errors.

The aforementioned Sunflower is an example of the self-supporting panel approach. Each petal is hinged at its inner edge to a central hub. When deployed, the petal is cantilevered at its inner edge and is independent of its neighbors. This version is about 30 feet in diameter. For larger collectors, more folds become necessary and adjacent panels must be structurally joined. Some possible concepts are discussed in Reference 7. An example is shown as figure 2. Of course, the objective of these designs is to enable automated deployment. The more in-space attachments that are required, the greater is the complexity of the deployment apparatus.

Deployable configurations using nearly hexagonal panels are possible as discussed, for example, in Reference 8. By using some secondary folds, a seven-hexagon collector about 12 meters in diameter can be stowed in the shuttle cargo bay. If an Aft Cargo Carrier were avallable, almost 20 meters in aperture would be possible. (See Reference 9.)

While many practitioners are convinced that the self-supported-panel approach can yield the accuracies necessary for even high concentration ratios and large sizes, it is the conviction of the authors, based on flight hardware experience, that the unit cost of this technique gets prohibitively large for large sizes. The ideas are illustrated qualitatively in figure 3 . The unit cost of the self-supporting-panel approach increases as collector size 
increases because of the added complexity of the deployer and, more importantly, the cost of the high precision necessary to maintain required accuracy. In addition, the cost of testing and proving the accuracy of the system on the ground becomes high. There is, in fact, a practical limit on the possible size of this approach.

On the other hand, the truss-supported approach costs more for small aperture sizes because of its increased complexity and the costs of assembling in orbit. But when the size increases, the unit cost decreases because much of the additional cost of the truss support is not very size dependent. The inherently stiffer structure with greater depth is less sensitive to fabrication errors and can be tested and measured on the ground more easily. The result is that much larger apertures are made practical by the truss-support approach. In addition, that approach could be less expensive even in the smaller size range considered in this paper, if high concentration ratios (approximately 2,000) are required. This may be particularly valid for the Space Station if manned or robotic assembly becomes a routine matter so that the costs of such assembly were reduced.

Examples of the truss-support concept are shown in Figures 4 through 7 taken from Reference 10, Figure 8 from Reference 11 and Figures 9 through 11 from Reference 9. They include a large range of implementation approaches from automated deployment, through assembly with a remote manipulator, to extravehicular assembly of individual panels to the support truss. Most are unproven but all are reasonable and obey the laws of physics.

The approach in Figure 4 is to attach panels to a previously deployed support truss. In Figure 5, the truss is attached to the panel before launch. The units are deployed and assembled to their neighbors on orbit.

An automated deployment scheme is shown in Figure 6 and 7 . In this approach, an intelligent robotic deployer expands each truss module and attaches it to its neighbors as needed. The modules are hinged together so that the number of attachments is minimized and the hinges help to guide the automated construction.

A very simple deployable concept is shown in Figure 8 . This approach is a descendant of the Extendible Support Structure which was flight-proven on the 
Synthetic Aperture Radar on the SEASAT spacecraft. Note that the panels nest when packaged so that deep dishes can be stowed efficiently.

Figures 9,10 and 11 show an assembly-intenstve approach where large pane 1 modules with pre-attached stowed support-truss segments are carried to orbit in the After Cargo Carrier. Each module is removed from the ACC, deployed and assembled. Collector areas of 300 square meters are possible with this approach.

Masses - The mass of the support structure can vary from zero with the self-stiffened panels to as much as the panel mass. For film reflectors, the support structure mass is equal to that of the reflector if a frontal membrane is used and will be many times greater (perhaps ten $\mathrm{kg} / \mathrm{m}^{2}$ ) if electrostatic pressure is used.

\section{Mounting Structure}

The collector must be positioned and oriented accurately with respect to the receiver which gathers the concentrated solar power. Furthermore, the combination must be pointed at the sun to an accuracy of a couple of milliradians. And the needed structure must not block too much solar energy.

For the inflatable collector, it is tempting to connect the frontal membrane to the receiver. This can lead to great difficulty with handing the heat which is re-emitted from the receiver cavity. Furthermore, such a configuration would requịre continuous pressurization. Hence, the usual concepts provide struts, perhaps inflated, connecting the receiver to the rim. Hard points for connections and means to distribute the point loads into the collector must be provided.

The self-supported panel concepts also are subject to the difficulty of providing hard points for attachment. On the other hand, attachment to the truss support structure is straightforward. Note, however, that space-use trusses are generally built of slender members so that care must be taken to ensure that the attachments cause no local moments to be applied to the joints. 
The attachment struts can be long, hinged members (see Figure 7, for example) or deployable or erectable beams. The choice is usually dictated by the texture of the collector, the type of spacecraft interface, and the stowage space avallable. The designer should avold placing the struts close to the mouth of the receiver lest the heat damage the structure.

\section{Selected Approach}

The approach selected for further study herein is the truss-supported panel concept. The main reason is its potential for constructing large collectors with high concentration ratios. Secondly, the Space Station is assumed to be able to provide on-orbit assembly in a routine fashion.

The cost-saving potential for the truss-supported approach has been pointed out. In addition, the structural integrity does not depend on the panels themselves. This not only allows more latitude in panel design but also simplifies the task of replacing panels on orbit. The higher natural vibration frequencies enabled by the deep truss also simplify operational procedures.

In the past several years, a great amount of effort has been devoted to the large infrared telescope which has an aperture of 20 meters. One of the attractive configurations is described in Reference 12 and is shown in Figure 12. The panels (in this case, weighing around ten $\mathrm{kg} / \mathrm{m}^{2}$ ) are mounted on a newly-invented synchronously-deployable truss structure called Pactruss. The deployment kinematics shown in Figure 13 have the property that the bays of the truss are very strongly coupled together. Thus, reliable synchronous deployment can be achieved with only a few actuators. A photograph of an early working model is shown in Figure 14.

The Pactruss deployable structure can also be configured in a square pattern. Such an example, in which the reflecting surface is offset, is shown in Figure 15. Reference 11 contains more detail on the square-cell geometry. 
SECTION 3

EFFECTS OF GEOMETRIC ERRORS ON CONCENTRATOR EFFICIENCY

Collectors of large effective concentration ratio are sensitive to small errors in the shape of the reflecting surface and to small errors in pointing the axis of the concentrator at the sun. The finite size of the sun's image also contributes to a reduction in the effective concentration ratio. Errors of a different sort arise when the reflecting surface consists of a mosaic of small independent spherical panels mounted on a common supporting truss structure. The effects of such errors on the effective concentration ratio of the concentrator are reported in this section. Note that the surface is assumed locally to be a perfect specular reflector in this section.

\section{Effects of Random Slope Errors \\ on the Effective Concentration Ratio \\ of a Continous-Paraboloidal Reflector}

The effect of geometric imperfections in the reflector surface on concentrator efficiency is a topic which has been studied by many investigators (see, for example, Reference 13). The objective of the study presented herein is to focus on the effects of those imperfections which are of particular importance in the structural design of precision reflector surfaces and supporting structures.

Consider first a perfect paraboloidal reflector with axiz $z$, as shown in Figure 16. The reflecting surface is described by the equation

$$
z=\frac{r^{2}}{4 F}
$$

where $r$ is the distance from the $z$-axis to a point on the surface, and $F$ is the focal length, or distance from the origin 0 to the center of the receiver $R$. For purposes of analysis, the receiver is assumed to be a circular disk of diameter $d$ and oriented orthogonal to the z-axis as shown in the 
figure. The diameter of the frontal projection of the paraboloidal reflector is $D$.

For a reflecting surface defined in this way, any incident ray parallel to the z-axis and intersecting the reflecting surface at a point $q$ will reflect into a ray which intersects the receiver at point $R$. However, the rays from the sun incident at $q$ are not all parallel to the z-axis since the sun's image is a disk in the sky subtending an angle $2 \varepsilon_{s}$. At a distance of approximately one astronomical unit, the sun half-angle $\epsilon_{s}$ is

$$
\epsilon_{s}=4.5 \mathrm{milliradians}
$$

Thus, as reflected at a point $q$, the rays from the sun fill a right circular cone with apex angle $2 \epsilon_{s}$ and axis $q R$.

In order to capture all of the sun's rays reflected at $q$, the receiver diameter $d$ must be selected to be sufficiently large. However, $d$ should not be chosen any larger than necessary to capture all the sun's rays emanating from every point $q$ on the reflector of diameter $D$. To do so would reduce the geometric concentration ratio, defined as $C$ where

$$
C \equiv\left(\begin{array}{l}
D \\
2
\end{array}\right)^{2}=\frac{\text { Frontal Area of Reflector }}{\text { Area of Receiver }}
$$

and would allow needless power losses in radiation from the hot receiver. The task of selecting the proper $d$ for a given $D$ (or vice versa) requires consideration of the geometry of the intersection of the cone of reflected rays and the receiver disk. For this purpose, consider a planar coordinate system centered at $R$ and containing the receiver disk, as shown in Figure 17 . In this coordinate system, the receiver is a circle of diameter $d$ centered at the origin.

The image of sun's rays on the receiver plane is an ellipse. Let $\rho$ represent the distance from $R$ to $q$. Then the major and minor axes of the ellipse are $2 p \epsilon_{s} / \cos \theta$ and $2 p \epsilon_{s}$, respectively, where $\theta$ is the angle between $R q$ and $R 0$, as shown in Figure 16. Let the $u$ and $v$ axes be defined as parallel to the 
minor and major axes of the ellipse, respectively, as shown in figure 17. For a reflector surface without geometrical imperfections, the ellipse is centered at $R$. However, in the presence of small errors in the slope of the reflector surface at $q$, the ellipse is not concentric with the receiver disk. In that case, the center of the ellipse is translated a distance $u_{0}$ and $v_{0}$ where

$$
\begin{aligned}
& u_{0}=2 \rho a_{u} \cos \theta \\
& v_{0}=2 \rho a_{v} / \cos \theta
\end{aligned}
$$

where $\alpha_{u}$ and $\alpha_{v}$ are the slope errors at $q$ in the $u$ and $v$ directions, respectively.

If the sun's image lies entirely within the receiver disk, then all of the incident energy at $q$ is delivered to the receiver. However, in the more general case, a portion of the sun's image lies outside the receiver disk as shown in Figure 17, and only a fraction of the incident energy at $q$ is delivered to the receiver. If the sun's image on the plane of the receiver is assumed to have uniform density, this fraction is the ratio of the area of intersection of the ellipse and circle in Figure 17, to the area of the ellipse, and may be called the local "capture ratio" $\gamma$ at $q$. The spatial average $\bar{\gamma}$ of the local capture ratio over the projected frontal area of the reflector then may be used to define the "effective concentration ratio" $C_{\text {eff }}$ in terms of $C$ as follows

$$
c_{\text {eff }}=\bar{\gamma} c
$$

Note that $c_{\text {eff }}$ can be considered to be the number of "suns" concentrated at the receiver. It is generally smaller than the geometric concentration ratio $C$ defined in equation (3). It is $C_{\text {eff }}$ which is of primary importance in solar energy collection.

To investigate the effects on $c_{\text {eff }}$ of random errors in the slope of the reflector surface, consider the special case when $\alpha_{u}$ and $\alpha_{v}$ are independent Gaussian random variables with zero mean and identical standard deviation $\delta_{\alpha}$. In this case $\gamma$ is also a random variable, and so is $C_{\text {eff }}$, although $C$ is deterministic. The expected value $E\left[C_{e f f}\right]$ of $C_{e f f}$ is then determined by 


$$
E\left[C_{e f f}\right]=C E[\bar{Y}]
$$

In order to determine the expected value, it is necessary first to calculate $\gamma$ as a function of $a_{u}, a_{v}, \theta, F / D$, and $c$. Numerical evaluation of $\gamma$ by simple strip-theory quadrature was used to develop the results presented herein. Averaging $\gamma$ over the reflector, again by numerical quadrature, yields $\gamma$.

For the situation just described, numerical results for $E[Y]$ were obtained by an additional numerical quadrature of the equation

$$
E[\bar{\gamma}]=\int_{-\infty}^{\infty} \int_{-\infty}^{\infty} \bar{\gamma}\left(a_{u}, a_{v}, \frac{F}{D}, c\right) \frac{1}{2 \pi_{\delta}{ }^{2}} \exp \cdot\left\{\frac{-\left(a_{u}{ }^{2}+a_{v}{ }^{2}\right)}{2 \delta_{\sigma}{ }^{2}}\right\} d a_{u} d a_{v}
$$

To facilitate the quadrature, the domain of integration in equation (8) was mapped to the strip $\rho>0,0<\phi<\pi / 2$ by utilizing symetry and with the substitution

$$
\begin{aligned}
& a_{u}=\sqrt{2 \rho} \cos \phi \\
& a_{v}=\sqrt{2 \rho} \sin \phi
\end{aligned}
$$

Note that the domain becomes finite since $\bar{\gamma}$ is zero for large enough values of p. Results are shown in Figures 18 through 22 as plots of $C$ versus $E\left[C_{e f f}\right]$ for different rms slope errors $\sigma_{\alpha}$, each figure corresponding to a different value of $F / D$.

The effects of large $\sigma_{\alpha}$ are typified by the curves in Figure 18. For example, when $\sigma_{\alpha}=10$ milliradians, the results show that for $F / D=0.3$, it is not possible to obtain a mean effective concentration ratio larger than about 1700 , no matter how large a geometric concentration ratio $C$ is chosen. Furthermore, the same figure shows that an $E\left[C_{e f f}\right]$ of 2,000, for instance, requires a $C$ of about 3,000 when $\sigma_{\alpha}$ is 5 milliradians but only a $C$ of about 
2,250 when $\sigma_{\alpha}$ is 3 milliradians. For the range of $E\left[C_{e f f}\right]$ shown in the figure, the curves for $\sigma_{\alpha}=0$ and $\sigma_{\alpha}=1$ milliradian are indistinguishable. For $E\left[C_{e f f}\right]$ up to 2,000 , the values of geometric concentration ratio are essentially equal to $E\left[C_{\text {eff }}\right]$. Similar basic trends for other values of $F / D$ ranging from 0.4 to 0.7 are shown in Figures 19 through 22.

From the design viewpoint, it is most often the case that a predetermined value of $C_{\text {eff }}$ is required. Then the design is specified in an overall geometric sense by selecting the shape $F / D$ and geometric concentration ratio $C$. It is therefore useful to consider the results in the form of $C$ versus $F / D$ for various levels of surface error $\sigma_{\alpha}$, as shown in Figures 23 through 25 . Each figure corresponds to a different level of $E\left[C_{e f f}\right]$. These figures again show that a smalier $C$ is required for smaller $\sigma_{\alpha}$ than for larger $\sigma_{\alpha}$. However, the figures also show the sensitivity of the required $C$ to $F / D$. For example, when $E\left[C_{\text {eff }}\right]$ is required to be 2,000 , Figure 25 shows that an optimal choice of $F / D$ exists. When $\sigma_{\alpha}$ is 5 milliradians, the curve displays a minimum $C$ at an $F / D$ of about 0.45. For values of $F / D$ substantially different from this optimal value, a significant increase in $C$ results. However, for smaller values of rms surface error, the optimum value of $F / D$ increases, although the curves show very little sensitivity to reasonably large deviations from the optimal F/D.

For desired mean effective concentration ratios up to 2,000, the surface slope error can be three milliradians without noticeable penalty for $F / D \cong$ 0.5 .

\section{Effects of Location, Orientation and Size of Nominally Square Spherical Panels on the Effective Concentration Ratio}

The application of large solar concentrators in space presents some difficulties in transportation. One practical approach to overcoming such difficulties is to erect the concentrator in space as a mosaic of paneis attached to a supporting truss structure. For such a panel design, obvious simplifications are gained by making the panels identical in shape, thus deviating from the desired paraboloidal surface. Near the center of a paraboloid, a very close approximation to the surface is provided by a sphere of radius $2 \mathrm{~F}$, where 
$F$ is the focal length of the parabolold. However, at points far from the center, such spherical panels may not match the paraboloidal surface very well. In order to minimize the degradation in effective concentration ratio caused by the use of spherical panels, various strategies for the location and orientation of the panels may be considered.

In order to effectively assembly a mosaic of panels without either excessive overlap or large holes, consider each panel to have a plan form which is a square of side length "a," as shown in Figure 26. Furthermore, let the radius of curvature of the panel be $2 \mathrm{~F}$.

Consider an $x, y, z$ coordinate system with the $z$-axis corresponding to the reflector axis, and with origin 0 at the center of the reflector surface, as shown in Figure 27. The center of the receiver $R$ is located a distance $F$ along the z-axis.

Let the panel be oriented so that for small angles of tilt, its projection onto the $x, y$ plane has sides which are always parallel to the $x, y$ coordinate directions, as shown in the figure. Furthermore, let the panel center be located at $c$, and let the projection of $c$ onto the $x, y$ plane be $c^{\prime}$. Then the points $0, R, c$ and $c^{\prime}$ lie in a plane containing meridional. lines of the reflecting surface, and labelled "meridional plane" in the figure. The meridional plane makes an angle $\psi$ with the $x$-axis. Let the angle between $c R$ and the $z$-axis be $\theta_{c}$, as shown in the figure.

Consider a point $p$ on the panel surface and also on the meridional plane. The location of $p$ is determined such that an incident ray at $p$ which is parallel to the z-axis reflects into a ray which intersects the receiver at its center, $R$. The distance from $R$ to $p$ is $\rho_{p}$, and the angle between the ray $p R$ and the $z$-axis is $\theta_{p}$, as shown. In this case, the local normal to the panel surface at $p$ makes an angle $\theta_{p} / 2$ with the z-axis.

For given values of $\rho_{p}, \theta_{p}$ and $\psi$, the center $s$ of the generating sphere from which the panel surface is derived may be located from the equations 


$$
\begin{aligned}
& \left(\frac{x_{s}}{F}\right)=\left[\left(\frac{\rho_{p}}{F}\right) \sin \theta_{p}-2 \sin \left(\frac{\theta_{p}}{2}\right)\right] \cos \psi \\
& \left(\frac{y_{s}}{F}\right)=\left[\left(\frac{\rho_{p}}{F}\right) \sin \theta_{p}-2 \sin \left(\frac{\theta_{p}}{2}\right)\right] \sin \psi \\
& \left(\frac{z_{s}}{F}\right)=\left[1-\left(\frac{\rho_{p}}{F}\right) \cos \theta_{p}+2 \cos \left(\frac{\theta_{p}}{2}\right)\right]
\end{aligned}
$$

where $x_{s}, y_{s}$, and $z_{s}$ are the coordinates of $s$. Note that $s$ lies in the meridional plane. The coordinates $x_{q}, y_{q}$, and $z_{q}$ of a generic point $q$ on the spherical surface are then governed by the equation

$$
\left[\left(\frac{x_{g}}{f}\right)-\left(\frac{x_{s}}{f}\right)\right]^{2}+\left[\left(\frac{y_{q}}{f}\right)-\left(\frac{y_{s}}{f}\right)\right]^{2}+\left[\left(\frac{z_{q}}{f}\right)-\left(\frac{z_{s}}{f}\right)\right]^{2}=4
$$

For a given receiver diameter $d$, the location of the panel center $c$ is determined in the following manner. First, consider temporarily locating the panel so that its center $c$ coincides with $p$. While in this position, the panel size $a$ is increased until the reflected rays from the worst case location on the panel (always a panel corner) contain sufficient error in direction that they first intersect the perimeter of the receiver disk. Clearly, the reflected rays from any smaller panel would all lie entirely within the receiver disk, but for a larger panel, a fraction of the reflected rays would miss the receiver altogether. The value of this maximum panel size is noted.

Next, the entire panel is rigidly rotated about the center $s$ of the generating sphere an incremental amount around an axis orthogonal to the meridional plane. During this motion, the panel sides remain parallel to the coordinate axes, but the center $c$ is translated along a meridional line away from $p$, as shown in the figure. In this new position, a new maximum panel size is determined by the procedure just described, and note is taken of the value. Then, the panel is similarly rotated through an additional incremental amount, and the process is repeated. The final location of the panel center is chosen as that location which permits the maximum possible panel size $a_{m}$ to be used. 
It is necessary at this point to discuss the nature of the errors included in the analysis of the reflected rays. First, the effects of the finite size of the sun's image in the sky, as measured by $\epsilon_{s}$, are included in a manner similar to that discussed in the previous section. In addition, a worst case mean pointing error in the alignment of the z-axis with the center of the sun is assumed. This angular pointing misalignment is $\epsilon_{p}$ in magnitude, and assumed for the fllustrations presented later to be

$$
\epsilon_{p}=0.15^{\circ}=2.62 \text { milliradians }
$$

When analyzing the effects of $\epsilon_{s}$ and $\epsilon_{p}$ on the reflected rays at any point $q$ on the surface, the local worst case for the direction of the pointing error is assumed. In addition to the errors just described, geometric errors are naturally introduced from the fact that the spherical reflecting surface deviates from the desired paraboloidal surface. However, the spherical surface is assumed to contain no random imperfections in this case.

The three remaining parameters which affect the maximum allowable panel sizes are $\psi, \theta$, and $\rho_{p}$. For panels located on a nominally square grid aligned along the $x, y$ coordinate directions, the panels will be located at various values of $\psi$. The effects of $\psi$ on the maximum allowable panel size will be presented later in the results.

For panels near the reflector axis, $\theta_{p}$ (and hence also $\theta_{c}$ ) will be small, but for panels near the outer rim, $\theta_{p}$ will be maximized for a given reflector. For spherical panels considered here, errors build up rapidly as $\theta_{p}$ is increased, and it is the panels near the rim of the reflector which govern the maximum panel size which may be used throughout. Consequently, only panels located near the rim are considered in the results presented later.

The distance $\rho_{p}$ from the receiver center $R$ to the point $p$ on the panel surface has a major effect on the maximum allowable panel size. In general, one would expect the optimal choice of $\rho_{p}$ for a given $\theta_{p}$ would be that value which results in a best fit of the spherical panel surface to the local surface of the paraboloid at $\theta_{p}$. However, selection of this best fit location is complicated by the fact that the spherical panel has a radius of curvature of 
$2 \mathrm{~F}$ which is the same in all directions, whereas the paraboloidal surface has different radij of curvature in the meridional and circumferential directions.

Three different approaches for selecting $\theta_{p}$ were investigated and are reported in the results. For a given $\theta_{p}$, these are (1) selecting $\rho_{p}$ such that the radius of curvature of the spherical panel matches the local meridional radius of curvature on a paraboloid, (2) selecting $\rho_{\mathrm{p}}$ such that the panel radius of curvature matches the local circumferential radius of curvature on a paraboloid, and (3) selecting $\rho_{p}$ as the geometric mean of the distances determined in (1) and (2) above. These three approaches result in the following selections for $\rho_{p}$, labelled to correspond with the cases outlined above

$$
\begin{aligned}
& \left(\frac{\rho_{p}}{F}\right)_{1}=\cos \left(\frac{\theta_{p}}{2}\right) \quad \ldots \text { "Meridional Curvature" } \\
& \left(\frac{\rho_{p}}{F}\right)_{2}=\frac{1}{\cos \left(\theta_{p} / 2\right)} \quad \ldots \text { "Circumferential Curvature" } \\
& \left(\frac{\rho_{p}}{F}\right)_{3}=1 \quad \ldots \text { "Geometric Mean Curvature" }
\end{aligned}
$$

Results are presented for each of these three approaches for determining $\rho_{p}$.

Shown in Figure 28 are results for the dependence of the dimensionless maximum panel size $\left(a_{m} / F\right)$ as a function of the angular location $\theta_{c}$ of the center of a panel. Different curves are shown for different values of $\psi$. All curves in the figure are for the case where $C_{\text {eff }}=2,000$ and $\left(\rho_{p} / F\right)=1$. Comparing the curves in the figure, each displays a local peak of $\left(a_{m} / F\right)$ at an intermediate value of $\theta_{c}$. For very small $\theta$ (corresponding to a high value of effective $F / D)$, the value of $\left(a_{m} / F\right)$ in all cases is very small because of the -effects of finite sun size $\epsilon_{s}$. For large $\theta_{c}$ (corresponding to small effective $F / D)$, the value of $\left(a_{m} / F\right)$ in all cases is again small, this time because the spherical shape of the panel differs considerably from the shape of a paraboloid surface. 
It is further noted in Figure 28 that the case where $\psi=0$ degrees (panel sides parallel to the coordinate axes $x$ and $y$ ) is somewhat of a best case in that significantly larger panels may be used than for other values of $\psi$. In many cases (although not in the case shown in the figure), the worst case occurs for $\psi=45$ degrees. This worst case therefore governs the size of panels in a practical design, and remaining results are presented for $\psi=45$ degrees.

The effects of pivoting the panel about the center $s$ of the generating sphere so that $c$ and $p$ are coincident are shown in Figure 29. Shown in the figures are curves for $\left(a_{m} / F\right)$ versus $\theta_{c}$ for the case where $\left(\rho_{p} / F\right)=1$ and $C_{\text {eff }}=2,000$. The solid line corresponds to results obtained by the pivoting maneuver previously described, while the dashed line indicates similar results obtained without pivoting so that $\rho$ and $c$ are always coincident. It is noted that the pivoting maneuver results in a significant increase in the maximum allowable panel size $\left(a_{m} / F\right)$.

Presented in Figures 30 and 31 are results which show the effects of the three different strategies for locating the panel center. Note that in both figures (corresponding to $C_{\text {eff }}=1,000$ and 2,000 , respectively, with a worst case $\psi=45$ degrees), the best choice is simply $\rho_{p}=F$, which corresponds to locating the panel centers on a sphere centered at the receiver center $R$. The worst case in both figures is the choice $\left(\rho_{p} / F\right)=1 / \cos \left(\theta_{p} / 2\right)$, which corresponds to locating the panel centers so that the panel radius of curvature $2 \mathrm{~F}$ matches the circumferential radius of curvature of the local paraboloidal surface. Note further that in each figure there exists a local peak for the curve $\left(a_{m} / F\right)$ versus $\theta_{c}$ corresponding to the best choice $\rho_{p}=F$. Hence, there exists an optimal effective $F / D$ for each case. In the case where $C_{\text {eff }}=1,000$ shown in Figure 30 , the peak occurs at $\theta_{c} \cong 1$. In the case where $C_{\text {eff }}=2,000$ shown in Figure 31 , the peak occurs at $\theta_{C} \cong 34$ degrees, corresponding to $F / D \cong 0.89$.

The foregoing results neglect the effects of surface slope errors in addition to the pointing error of 0.15 degrees. Inasmuch as the analysis is based on the requirement that the entire reflected beam from all parts of the reflector is captured by the receiver, it is probable that the losses due to 
one to three milliradians of slope error would be small. The combined analysis is relegated to the future.

\section{Summary of Findings}

Based on the analyses presented in this section, the conclusion is that, for continuous paraboloidal reflectors with mean effective concentration ratio up to 2,000, an rms surface slope error as large as three milliradians may be tolerated without significant loss in concentrator efficiency. Furthermore, the optimal value of $\mathrm{F} / \mathrm{d}$ in this case appears to be slightly less than 0.5 .

For reflectors consisting of a mosaic of individual spherical panels of side length $a_{m}$, the panel size may be as large as $F / 10$ without significant loss in efficiency for mean effective concentration ratios up to 2,000. These conclusions are based on an analysis of the effects of errors due to aberrations in the mosaic reflector surface, finite sun size, and an overall concentrator pointing error of 0.15 degrees.

Furthermore, the results should not be very sensitive to moderate increases in the pointing error due to local distortions, because the results are based on an assumption of complete capture in the worst-case direction for the worst-case corner on the worst-case panel in the mosaic. Therefore, only a moderate loss in captured energy would be incurred on a small portion of the surface area if a larger pointing error were present. 
SECTION 4

EXAMPLE OF TRUSS-SUPPORTED CONCEPTUAL DESIGN

The analysis in Section 3 establishes that high-efficiency, highconcentration ratio solar concentrators can be constructed of identical panels that are properiy placed and oriented. In particular, a concentration ratio of 2,000 is comfortably achieved with identical square panels having a size of one-tenth the concentrator diameter located at a distance from the receiver equal to the diameter. The conceptual design described in this section is based on those ratios.

The choice of square facets for the Pactruss enables significant simplification in the design of the truss. Let the coordinate along the solar axis be $z$ and the shape of the truss surface to which the panels are mounted be given by the form

$$
z=f(x)+f(y)
$$

The savings in joint similarity and tooling costs are obvious.

Of course, the paraboloidal surface obeys the foregoing equation. A spherical shape does not, but the departures for the proportions considered herein are small. Furthermore, although the central points on the panels are supposed to be equidistant from the receiver, the outer corners, which are assumed to be mounted discretely at the truss nodes, do not lie on a sphere. A computer program (SOLARPAC.C) for calculating the function $f(x)$ for chosen proportions of the panels and truss, the focal length, and the number of panels is included in the Appendix. This program considers panels oriented along the $x$-axis (or $y$-axis) whose central points are located on a sphere and whose tilt is adjusted so that the ray reflected from the central point is directed to the center of the receiver entrance. (The geometrical changes required to account for the improved accuracy discussed in Section 3 are small and are ignored here.) 
In using SOLARPAC, the panel size must be chosen smaller than the strut length. Otherwise, interference occurs between the corners of adjacent panels along the $x=y$ direction. The resulting panel geometry is shown in Figure 32. The proportions are:

Panel size $=2.0$ meters

Strut length $=2.15$ meters

Focal length $=21.5$ meters

Note the close meeting of the outer panel corners near $x=y$ and the gaps elsewhere.

Some consideration was given to the use of an offset concentrator geometry in order to avoid problems with blockage. But the loss of efficiency involved in such an approach was considered to be unacceptable. This is the price to be paid for being able to use the identical-panel concept. Note that the blockage problem is avolded by providing a slot along the concentrator centerline in which mounting structure and radiator panels can be located. The truss structure carries through but the panels are omitted.

The overall conceptual design as applied to the Space Station is shown in Figure 33. The concentrator is composed of 86 identical two-meter-square panels, fabricated with a spherical radius of curvature of 43 meters. The capture area is 328 square meters. Each panel is mounted directly to a Pactruss node at its outermost corner; short standoffs connect it to the other nearby nodes. The support truss is attached to the receiver by means of an erectable mounting truss made of the same diameter and similar fittings as those of the primary Space station truss. The mounting truss has the same five-meter dimensions in one plane but has a depth of only two meters in order to avoid blockage. The mounting truss is connected to the Space Station Beta joint with a transition truss which has a variable geometry so that fine pointing is enabled. The attachment location is selected so that the center of gravity of the entire solar power generator is in line with the transverse beam center.

The radiator is closely coupled to the receiver-converter in order to minimize the length of heat lines. The radiator is also located in the slot and 
is supported against out-of-plane motion by thermal-expansion-tolerant joints with the support truss.

A possible scenario for constructing the new concept on the Space Station would be as follows:

(a) Erect the mounting truss from the Beta joint.

(b) Attach the stowed Pactruss to the mounting truss.

(c) Deploy the Pactruss using its self-contained joint actuators.

(d) Mount the reflector panels onto the Pactruss joints.

(e) Attach the receiver/converter to the mounting truss.

(f) Attach the radiator to the receiver/converter and the Pactruss.

The resulting configuration is stiff and efficient. Its free-free fundamental vibration frequency is estimated by the method in Reference 10 to be greater than ten Hertz. Its construction makes straightforward use of the assembly facilities which must be available on the Space Station. Estimates of the mass are as follows:

Panels: $\quad$ one-inch thick aluminum honeycomb with metallized graphite faces.

$344 \mathrm{~m}^{2}$ @ $2.0 \mathrm{~kg} / \mathrm{m}^{2}$ $688 \mathrm{~kg}$

Pactruss: one-inch diameter graphite tubes with 0.020 -inch wall. 2.0 joint factor. $2,430 \mathrm{~m} \odot 0.15 \mathrm{~kg} / \mathrm{m}$ $355 \mathrm{~kg}$

Connecting Truss: two-inch diameter graphite tubes with 0.060 -inch wall. 1.5 joint factor.

$449 \mathrm{~m} \odot 0.66 \mathrm{~kg} / \mathrm{m}$ $296 \mathrm{~kg}$

The total mass of the collector and the mounting structure is 1,339 kilograms for a weight density of $4.1 \mathrm{~kg} / \mathrm{m}^{2}$ based on a frontal area of 328 square meters. 
The stowage volume of the structural parts of the configuration is small. The volumes are estimated to be:

Panels: $\quad 86$ panels with three-centimeter depth per panel.

$2 \mathrm{~m} \times 2 \mathrm{~m} \times 2.6 \mathrm{~m}$ $10.4 \mathrm{~m}^{3}$

Pactruss: stowed size of 3-1/2 diameters per bay.

$1 \mathrm{~m} \times 1 \mathrm{~m} \times 4.3 \mathrm{~m}$

$4.3 \mathrm{~m}^{3}$

Connecting

Truss:: 100 struts of two-inch diameter, up to five long. 32 balls.

$0.5 \mathrm{~m} \times 0.5 \mathrm{~m} \times 5 \mathrm{~m}$

$1.3 \mathrm{~m}^{3}$

$17.0 \mathrm{~m}^{3}$ 
SECTION 5

CONCLUDING REMARKS

The study described herein needs to be extended to include more quantitative evaluation of the dependency between cost and required precision for the concentrator structure. The emphasis during this evaluation should be on a comparison between the self-supported and truss-supported panel concepts. In order for the comparison to be valid, detalled preliminary design, fabrication planning and test planning would be needed for several sizes in order to avoid overoptimistic estimates.

Continued investigation is needed for film-type collectors. In particular, a material is needed which will satisfy the long-life, highefficiency requirements of the Space Station. 


\section{REFERENCES}

1. Zipkin, Morris A. and Edwards, Russell N., eds: "Power Systems for Space Flight," based on a selection of Technical Papers from the American Rocket Society Space Power Systems Conference held at Santa Monica, California on 25-28 September 1962. Academic Press, New York, 1962.

2. Szego, George C. and Taylor, J. Edward, eds.: "Space Power Systems Engineering," based on a selection of Technical Papers from the American Institute of Aeronautics and Astronautics, Third Biennial Aerospace Power Systems Conference held at Philadelphia, Pennsylvania on 1-4 September, 1964. Academic Press, New York, 1964.

3. Thompson, Ramo Wooldridge, Inc.: "Sunflower Solar Collector," NASA Contractor Report CR-46, prepared under Contract No. NAS5-462. National Aeronautics and Space Administration, Washington, D.C., May 1964.

4. "Proceedings, Fifth Parabolic Dish Solar Thermal Power Program Annual Review" presented at Indian Wells, California on 6-8 December, 1983, JPL Publication 84-13. Jet Propuision Laboratory, California Institute of Technology, Pasadena, California, 1 March 1984.

5. Roschke, E. John; Owen, William A.; Menard, Wesley A. and Fujita, Toshio: "Trends in Concentrator, Receiver, and Storage Development for Terrestrial Applications," Space Power, NASA Conference Publication 2352, presented at a workshop held at NASA Lewis Research Center in Cleveland, Ohio on 10-12 April, 1984. Jet Propulsion Laboratory, California Institute of Technology, Pasadena, California.

6. Bernasconi, M. C. and Reibaldi, G. G.: "Inflatable, Space-Rigidized Structures: Overview of Applications and Their Technology Impact," preprint IAF 85-210, published for IAF '85, the 36 th Congress of the International Astronautical Federation held in Stockholm, Sweden on 7-12 October, 1985.

7. Archer, J. S.: "Advanced Sunflower Antenna Concept Development," presented at the Large Space Systems Technology (LSST) 1st Annual Technical Review held at TRW on 7-8 November 1979.

8. Ard, K. E.: "Design and Technology Study for Extreme Precision Antenna Structures," NASA Contractor Report CR-174861, prepared under NASA Contract No. NAS3-23249 for the National Aeronautics and Space Administration, Lewis Research Center, Cleveland, Ohio. Harris Corporation/Government Aerospace Systems Division, Melbourne, Florida, August 1985. 
9. Hedgepeth, J. M.; Mobley, T. B. and Taylor, T. C.: "Construction of Large Precision Reflectors Using the Aft Cargo Carrier," IAF-84-389, presented at the IAF ' 84 , the 35 th Congress of the International Astronautical Federation held in Lausanne, Switzerland on 7-13 October 1984.

10. Hedgepeth, John M.: "Support Structures for Large Infrared Telescopes," NASA Contractor Report No. 3800, prepared under NASA Contract No. NAS1-16923. National Aeronautics and Space Administration, Washington, D.C., July 1984.

11. Hedgepeth, John M.: "New Concepts for Precision Reflector Support Structures," IAF-85-208, presented at IAF 186, the 36th Congress of the International Astronautical Federation held in Stockholm, Sweden on 7-12 October 1985.

12. Swanson, P. N.: "A Lightweight Low-Cost Large Deployable Reflector (LDR), A Concept Study by the Jet Propulsion Laboratory," JPL D-2283. Jet Propulsion Laboratory, California Institute of Technology, Pasadena, California, 1985.

13. Jerke, J. M.; and Heath, A.R., Jr.: "Geometric Properties of a Modified Whirling-Membrane Solar-Energy Concentrator," NASA TN D-5859, Langley Research Center, Hampton, VA, June 1970. 


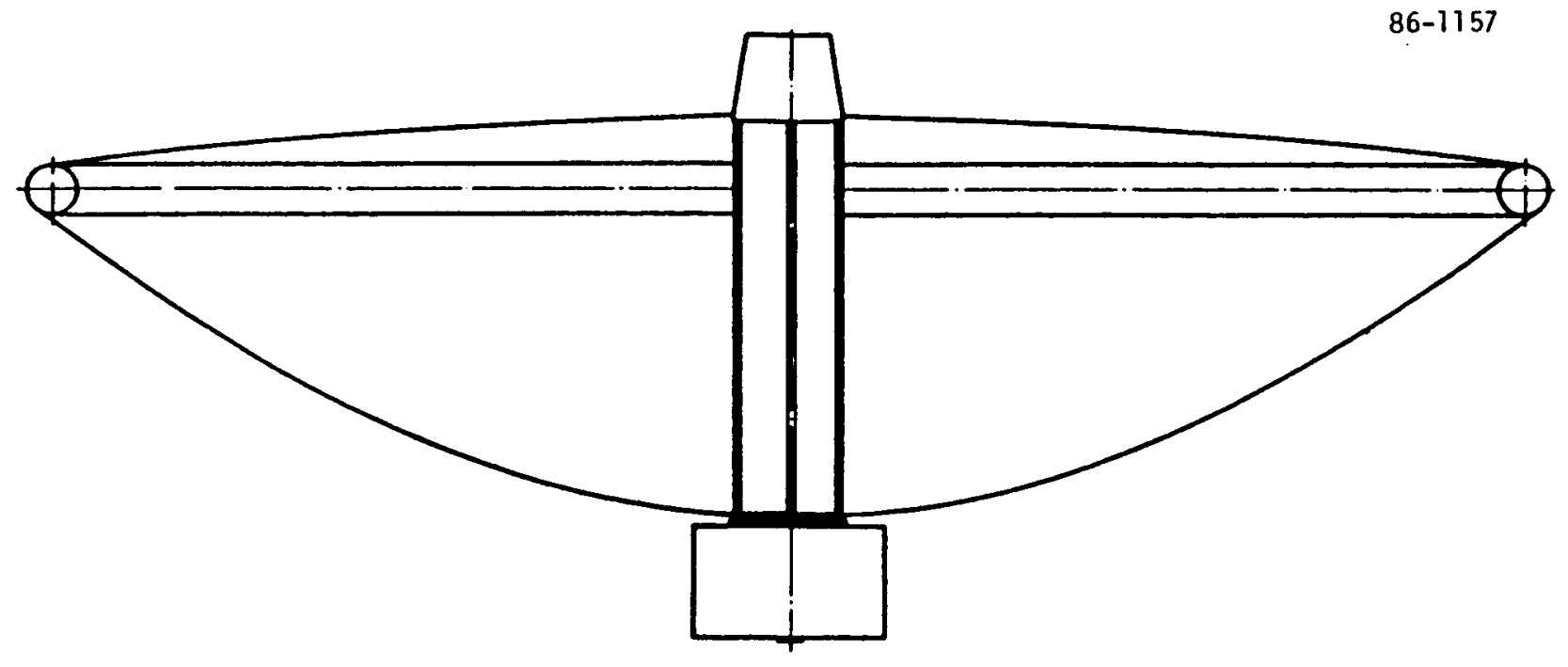

Figure 1a. Symmetric antenna reflector configuration (QUASAT - 20-m aperture).

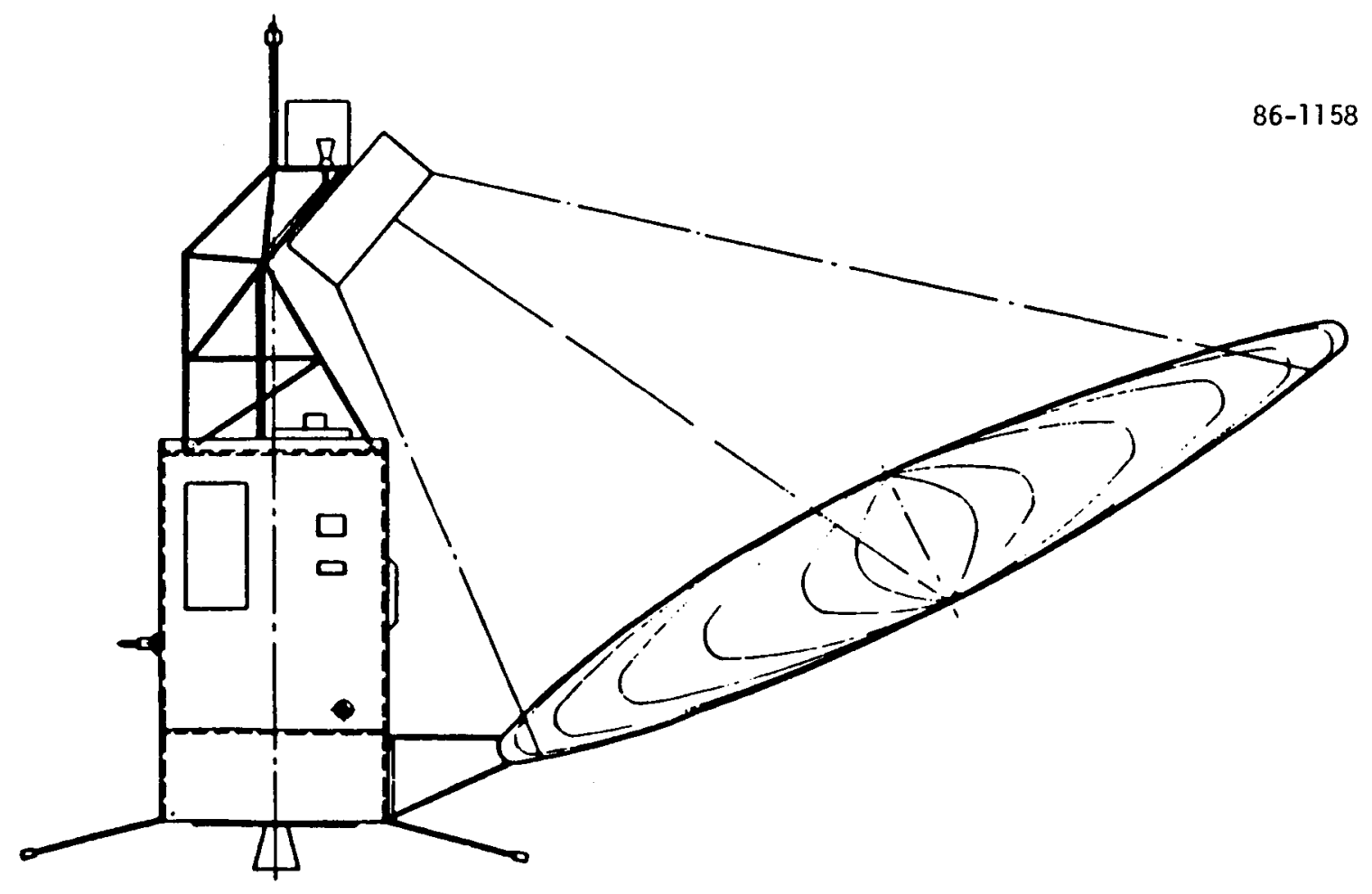

Figure 1b. Offset-fed antenna reflector configuration. 


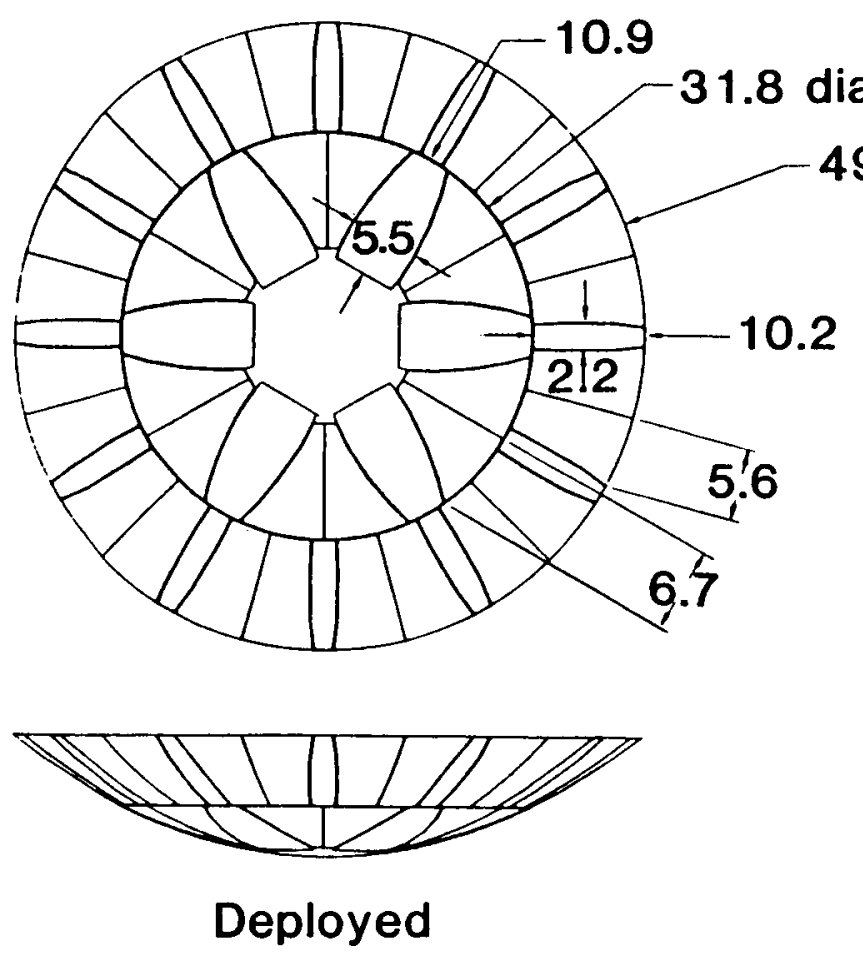

86-1156 49.2 ft. O.D.

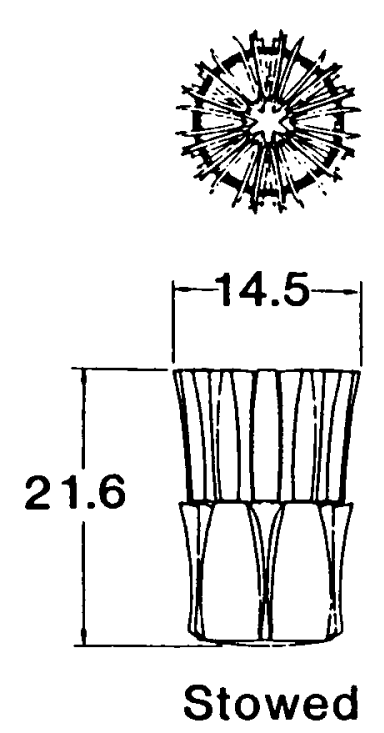

Figure 2. Main panel double ring configuration.

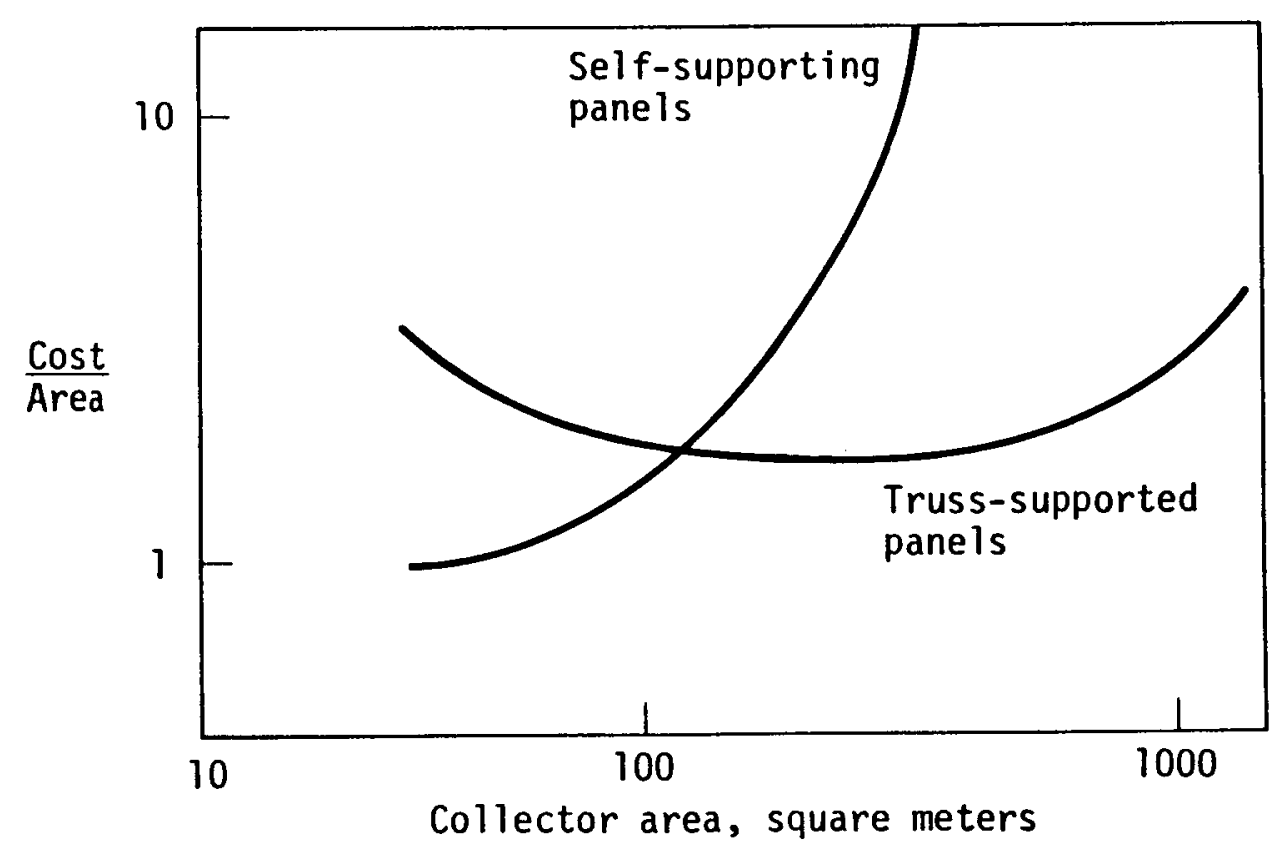

Figure 3. Qualitative comparative unit costs for panel-type solar collectors. 


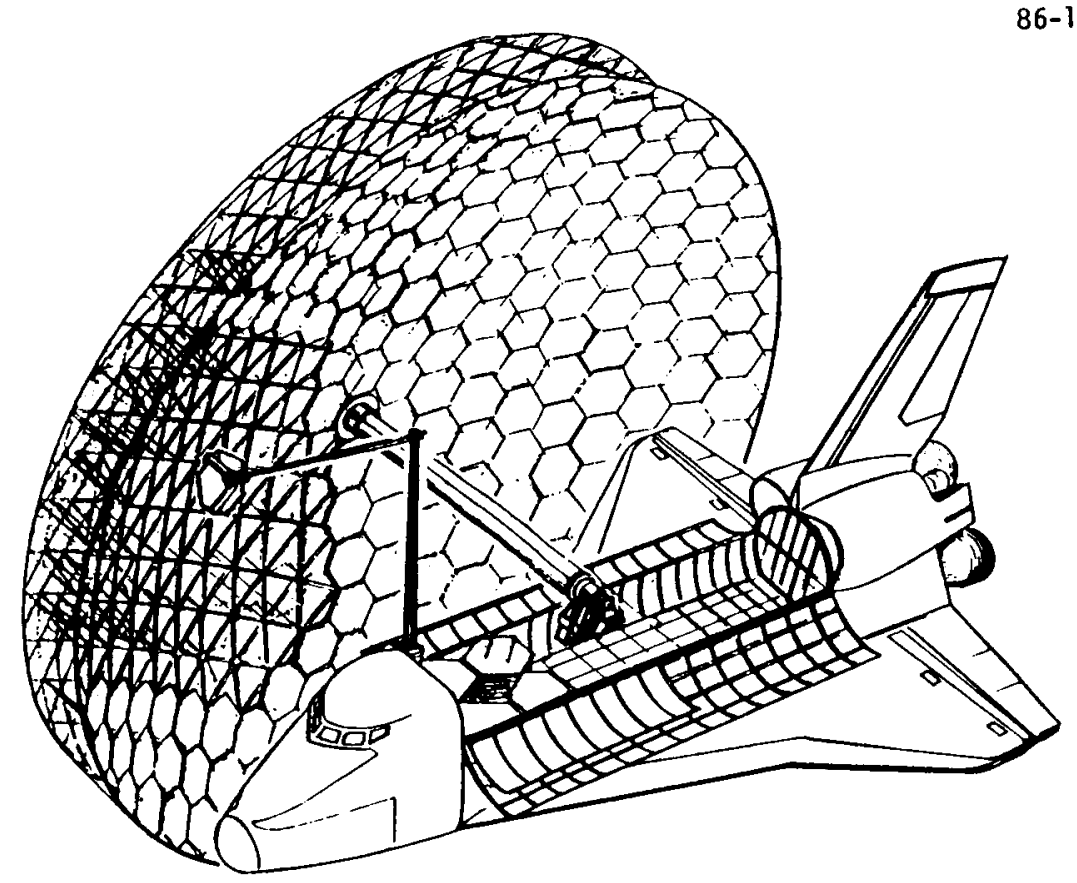

Figure 4. Mosaic reflector of spherical segments assembled by RMS from Shuttle.

$86-1161$

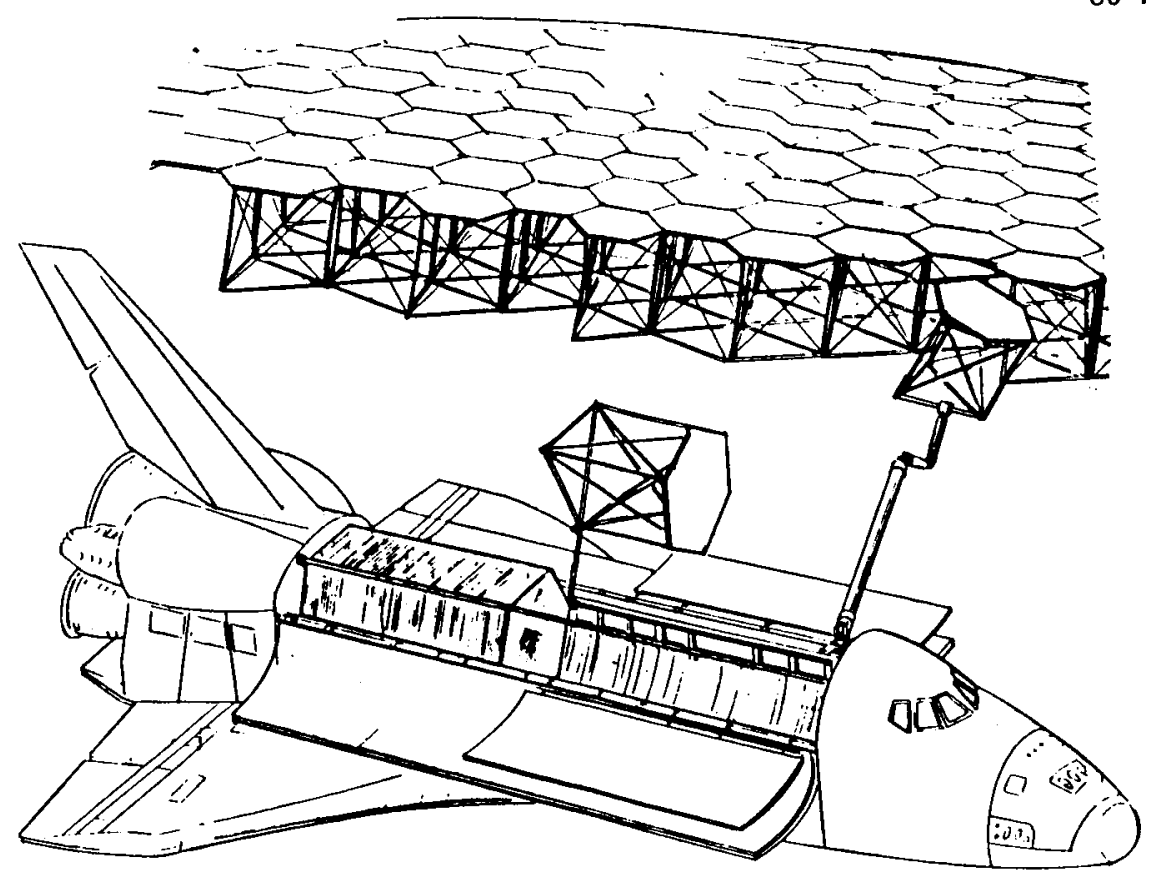

Figure 5. Assembly of integrated panel-truss modules. 


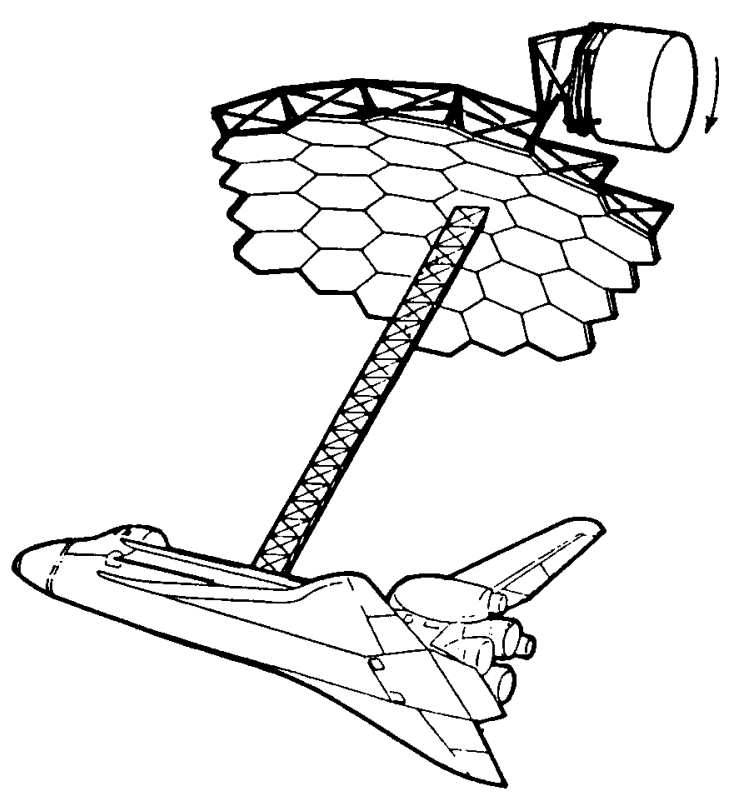

$86-1166$

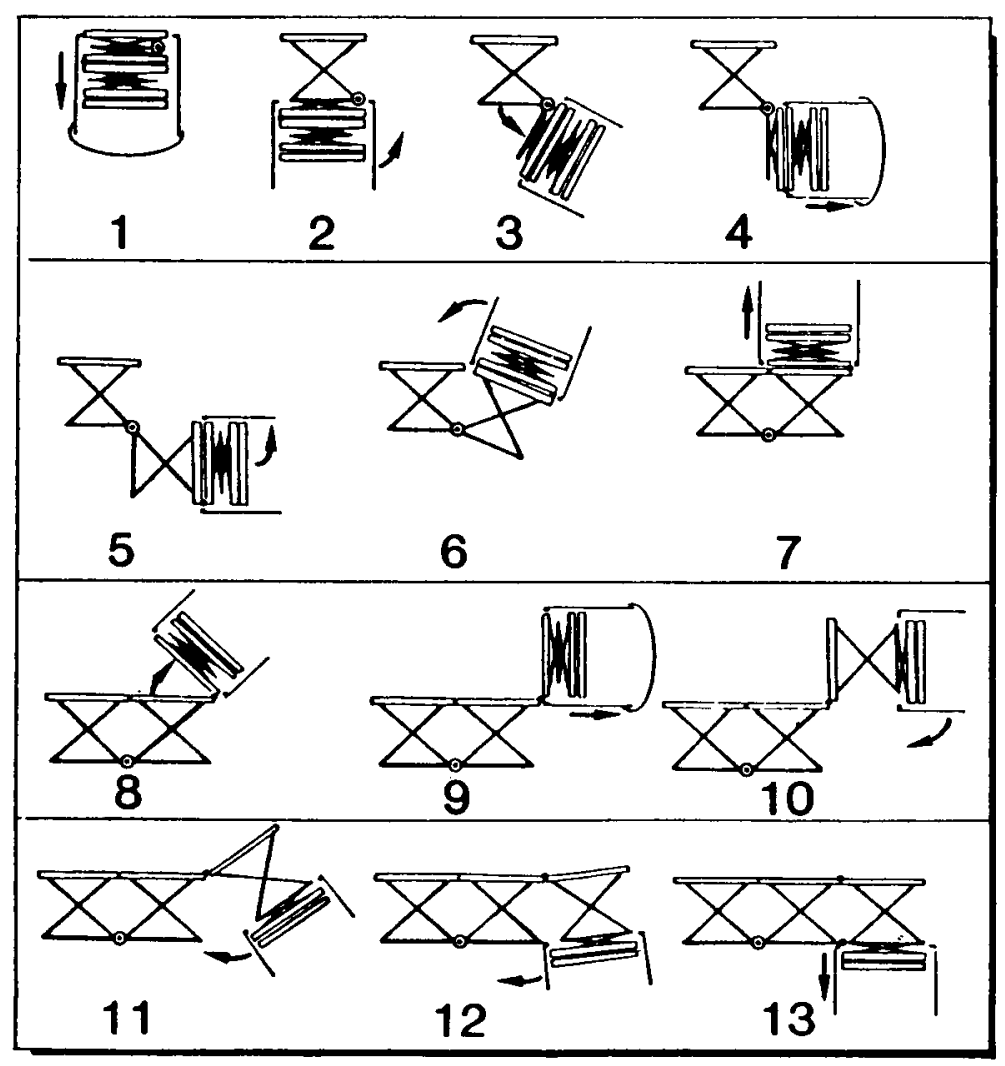

Figure 6. Sequential deployment of connected panel-truss modules. 
$n$
$\cdots$
1
$\infty$
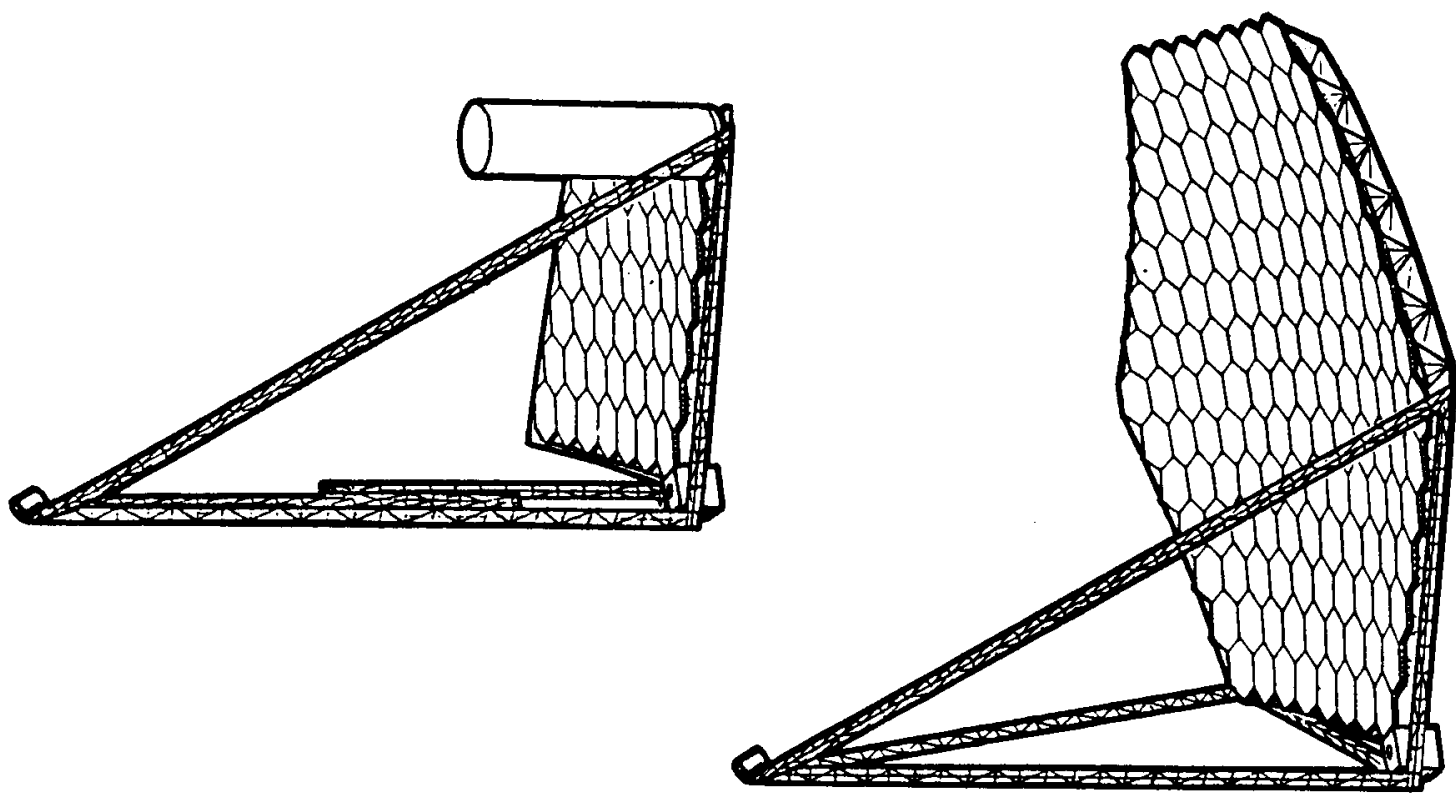

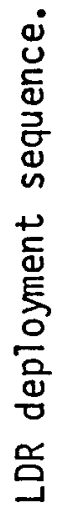
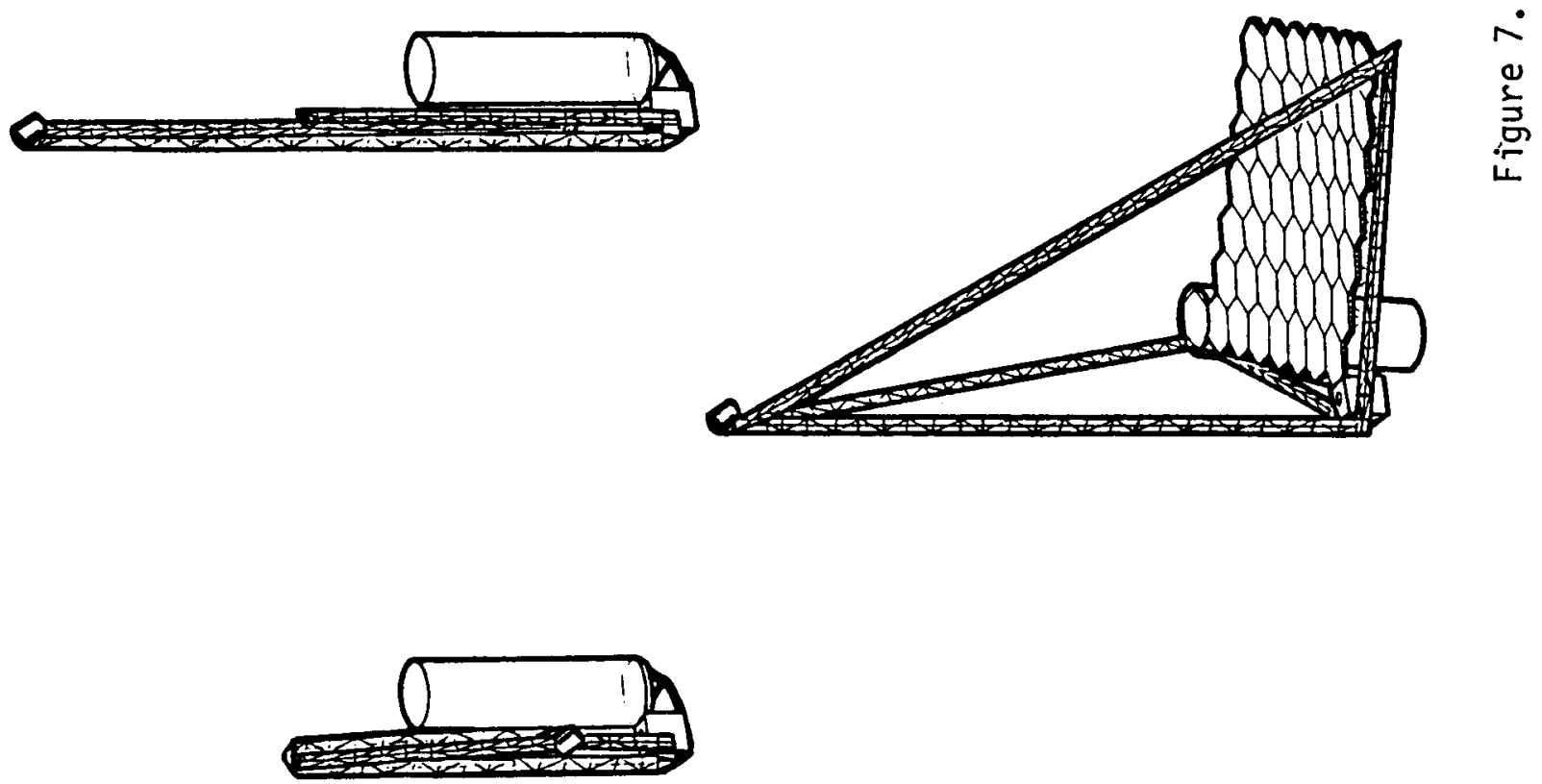
$n$
$\infty$
1
1
$\infty$
$\infty$
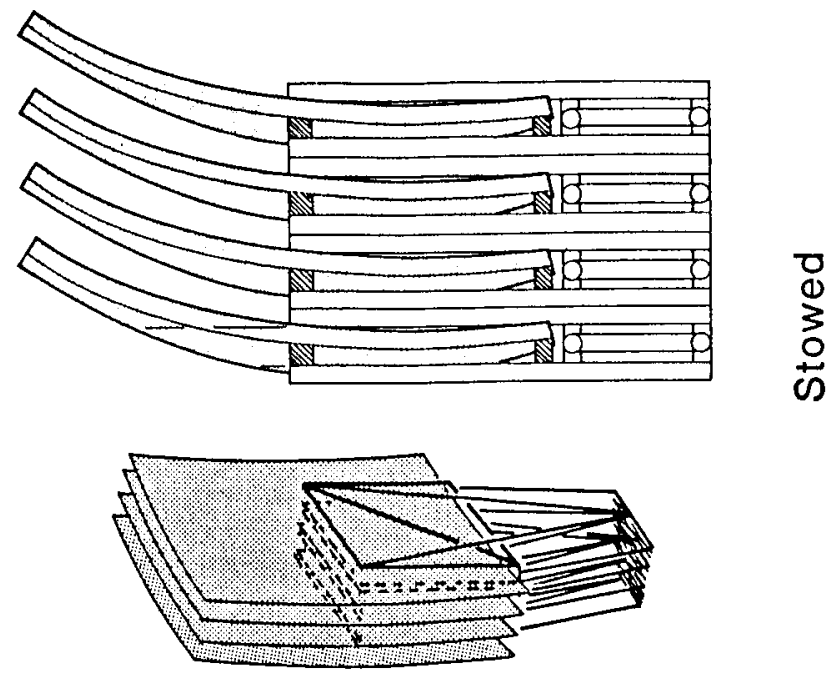

U
U
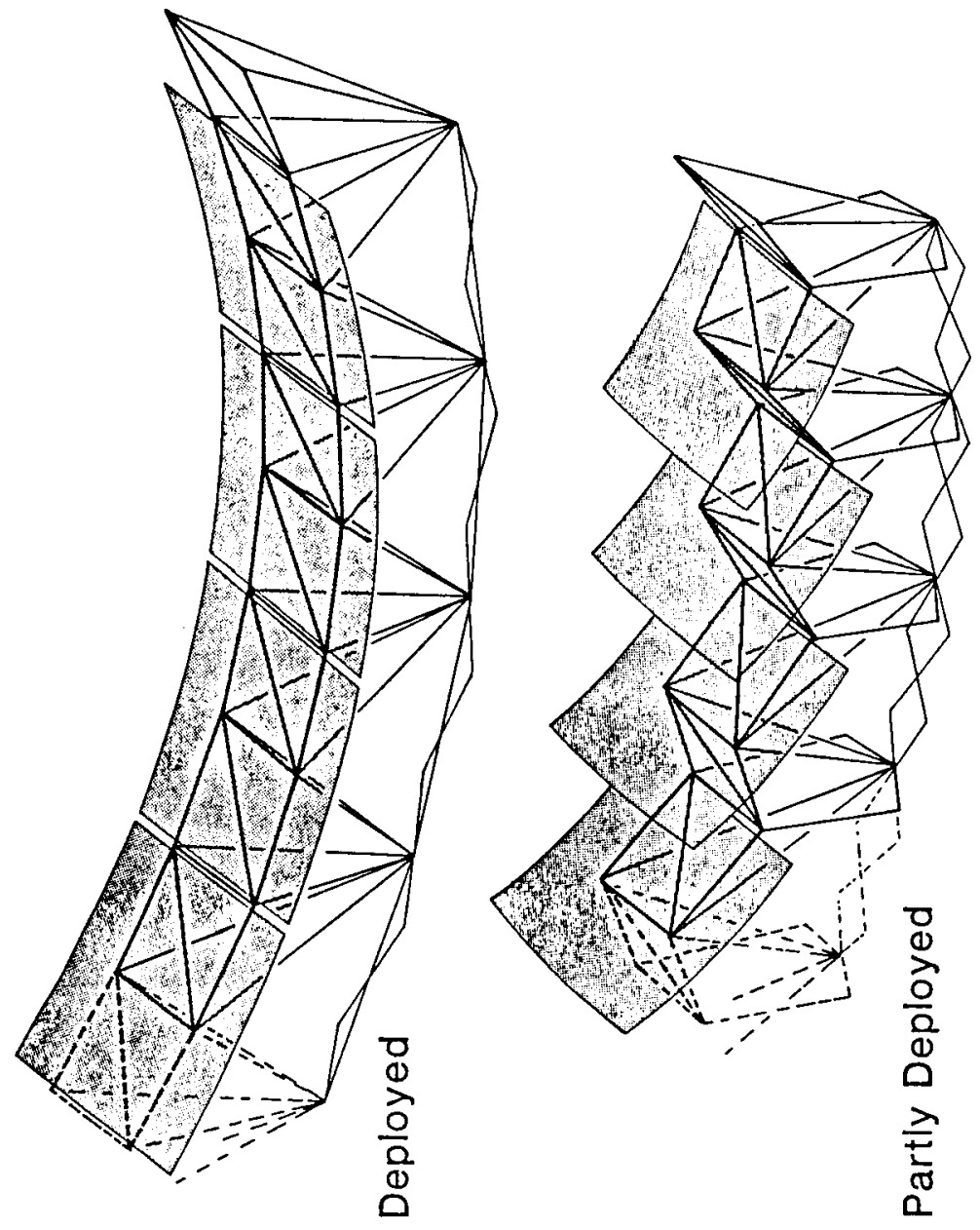

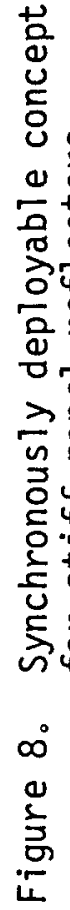




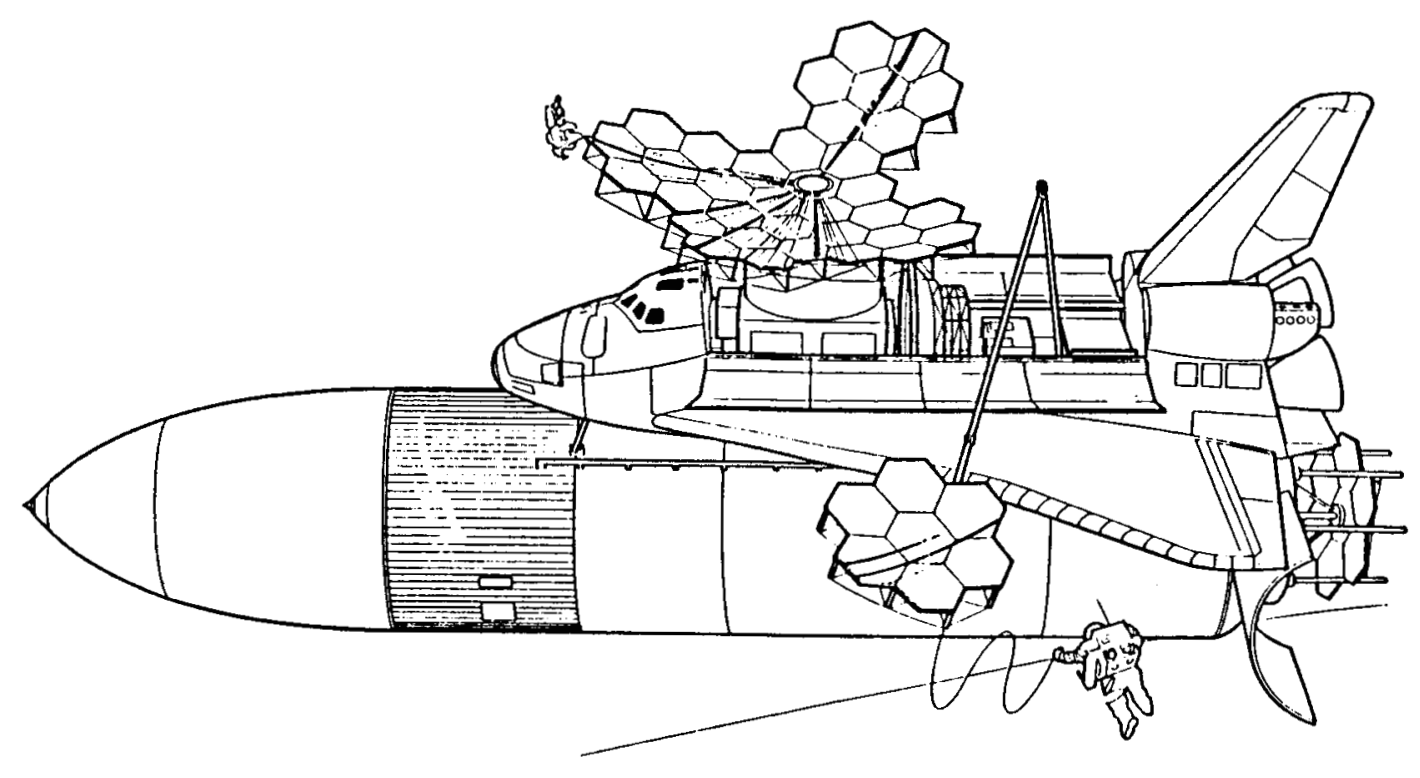

Figure 9. Assembly of the LPR infrared telescope..
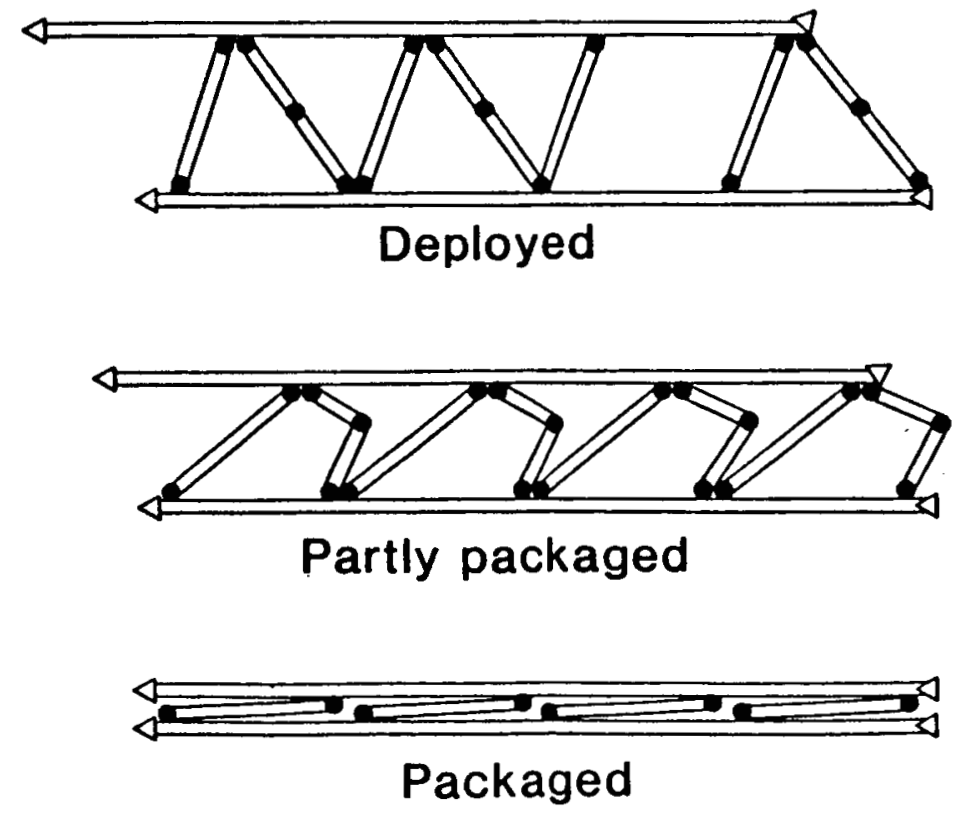

Figure 10. Deployment of preintegrated support-truss modules for the LPR infrared telescope. 


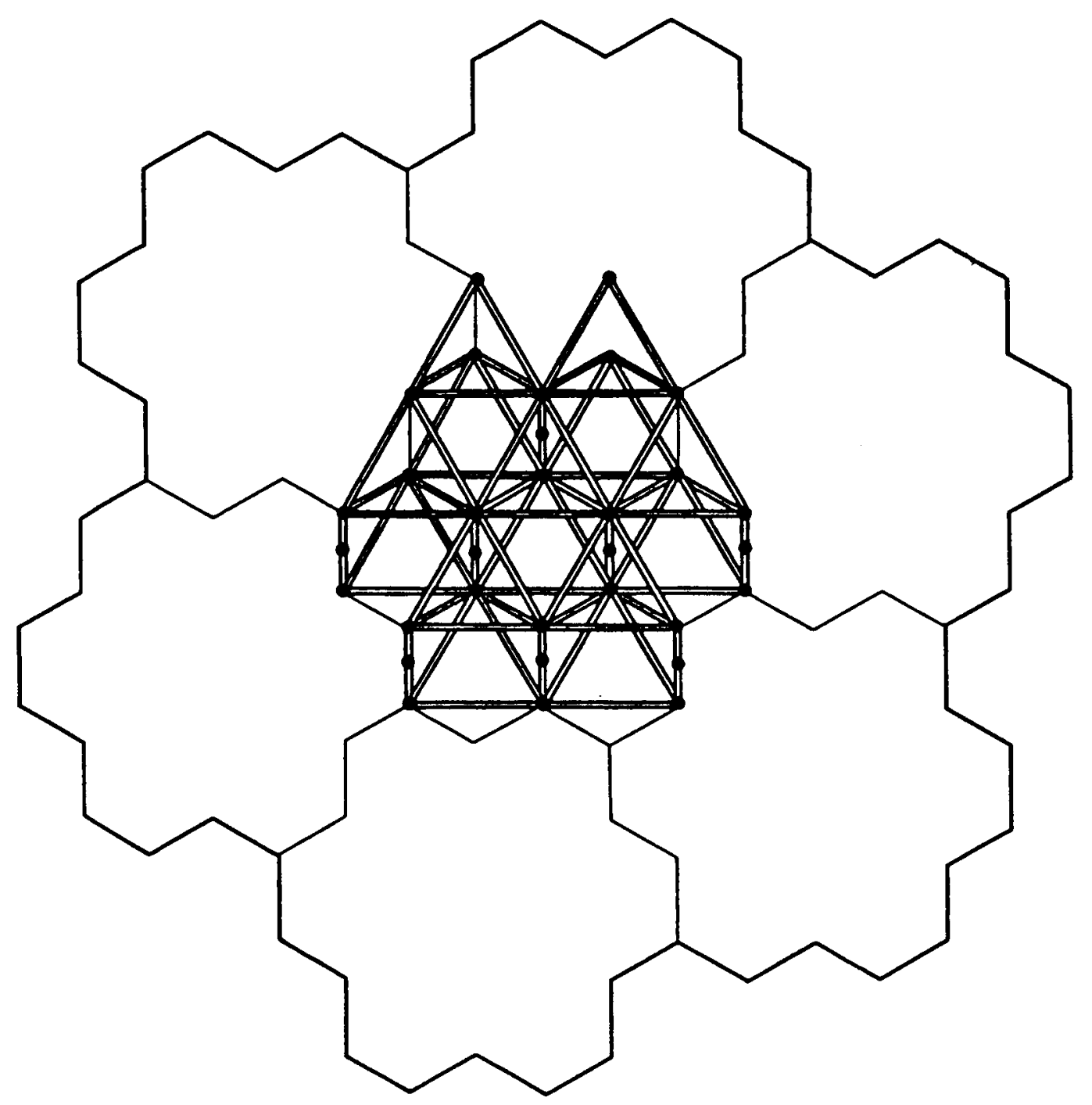

Figure 11. Support-truss for a seven-tile module of the LPR infrared telescope. 


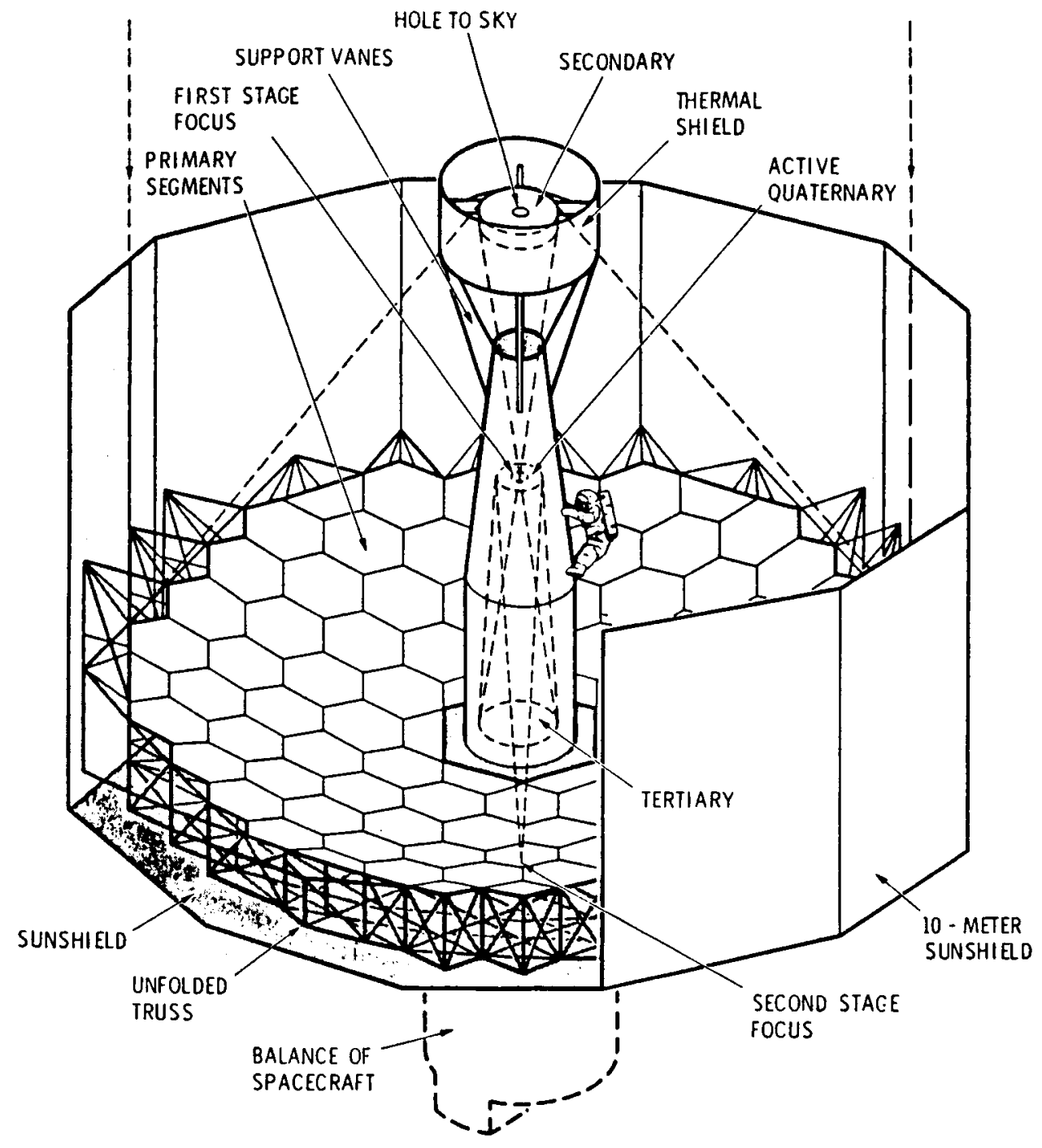

Figure 12. Application of Pactruss to the support structure for the primary mirror of the JPL low-cost LDR. 


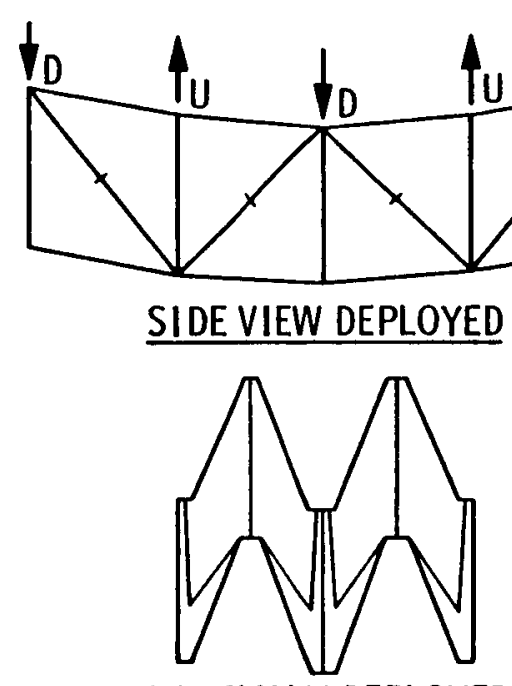

PARTIALLY DEPLOYED

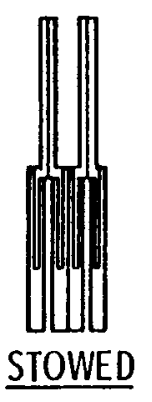

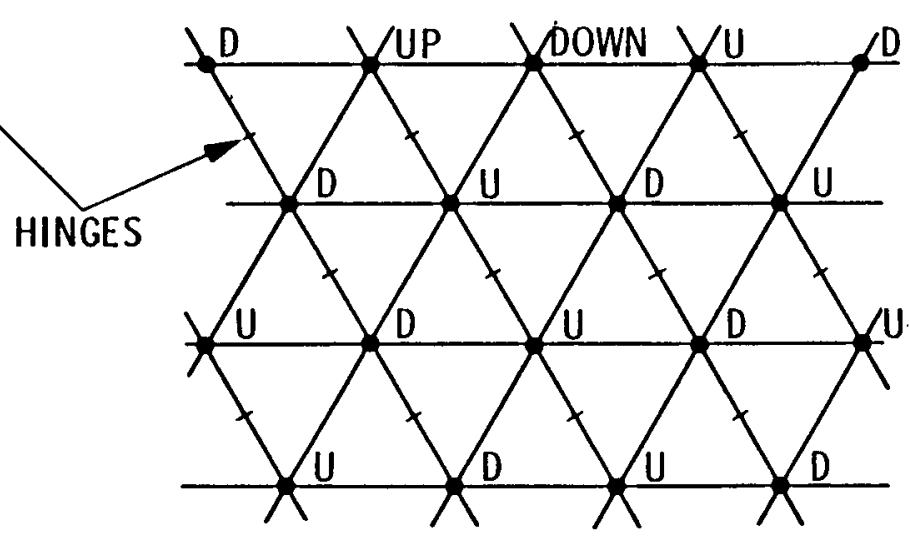

PLAN VIEW DEPLOYED

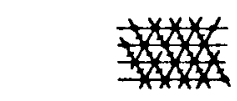

PLAN VIEW STOWED

Figure 13. Synchronously deployable Pactruss concept. 
of POOR QUALITY

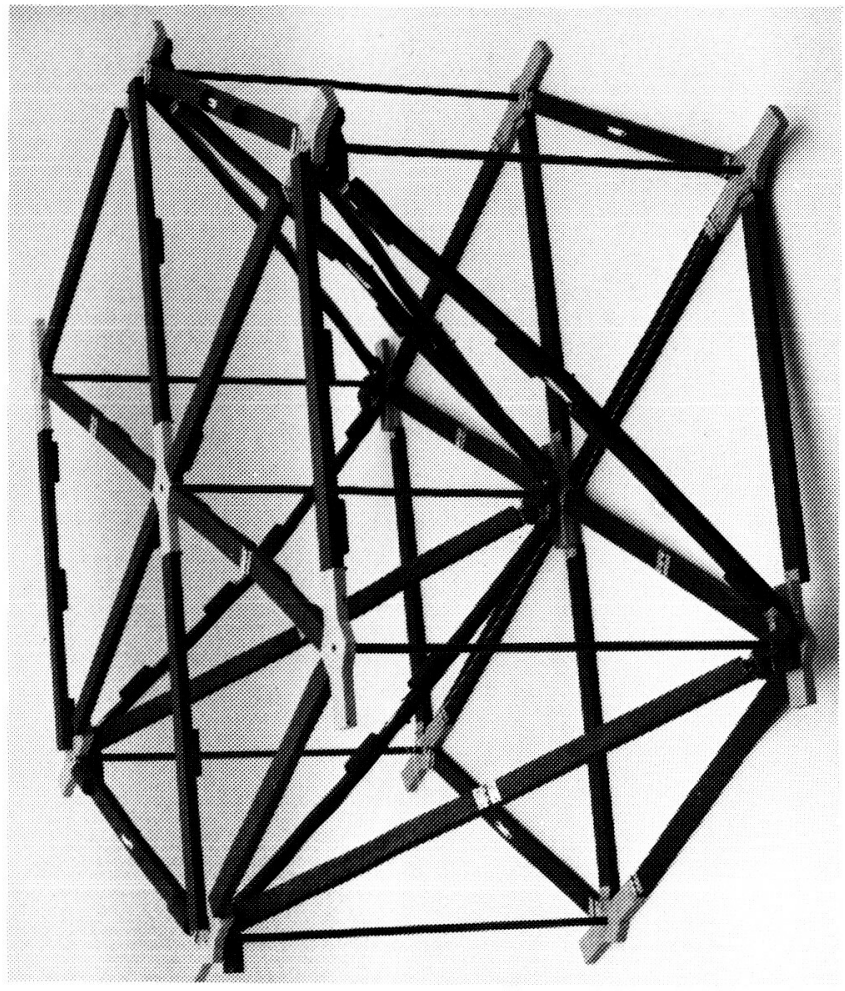

$m$
$\infty$
1
$\infty$
$\infty$

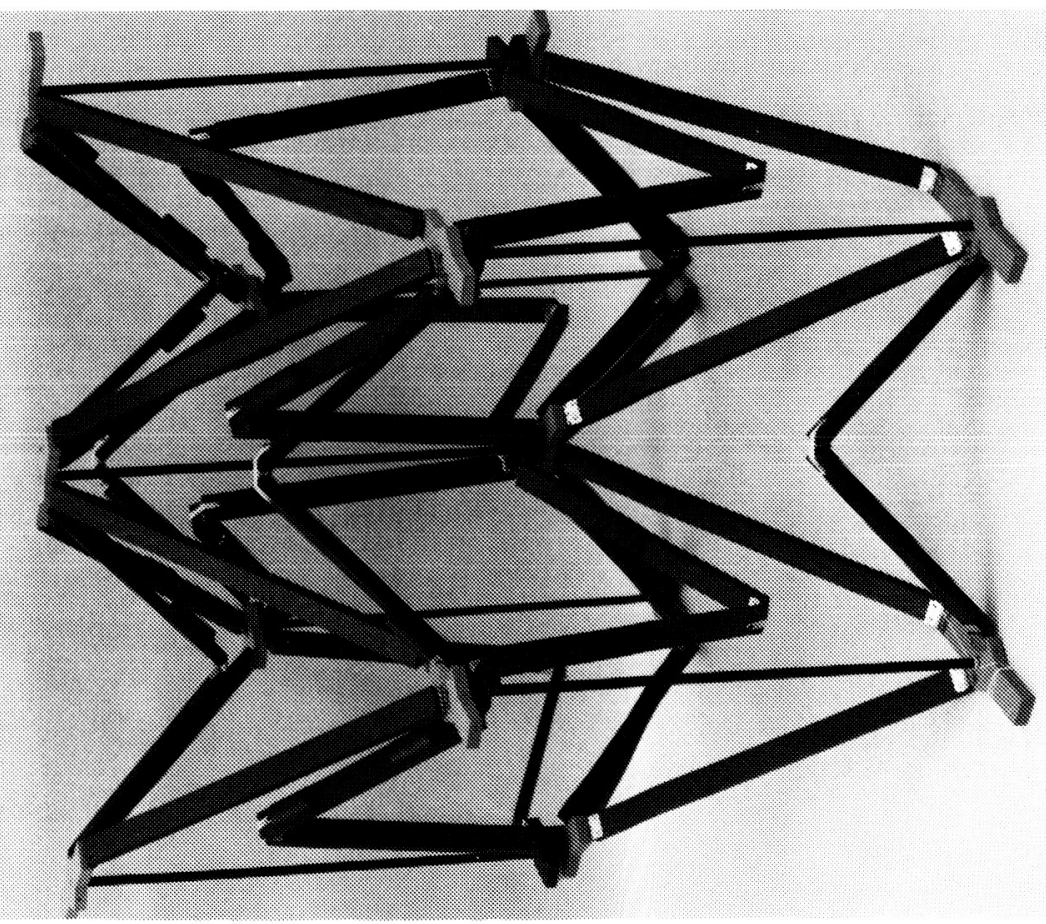

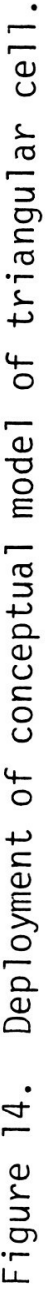

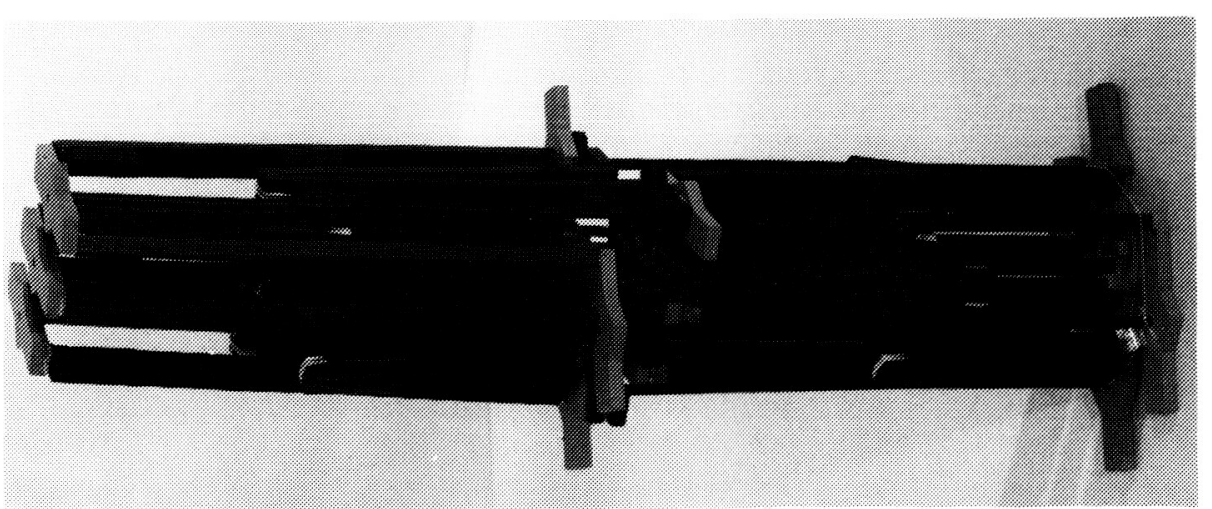



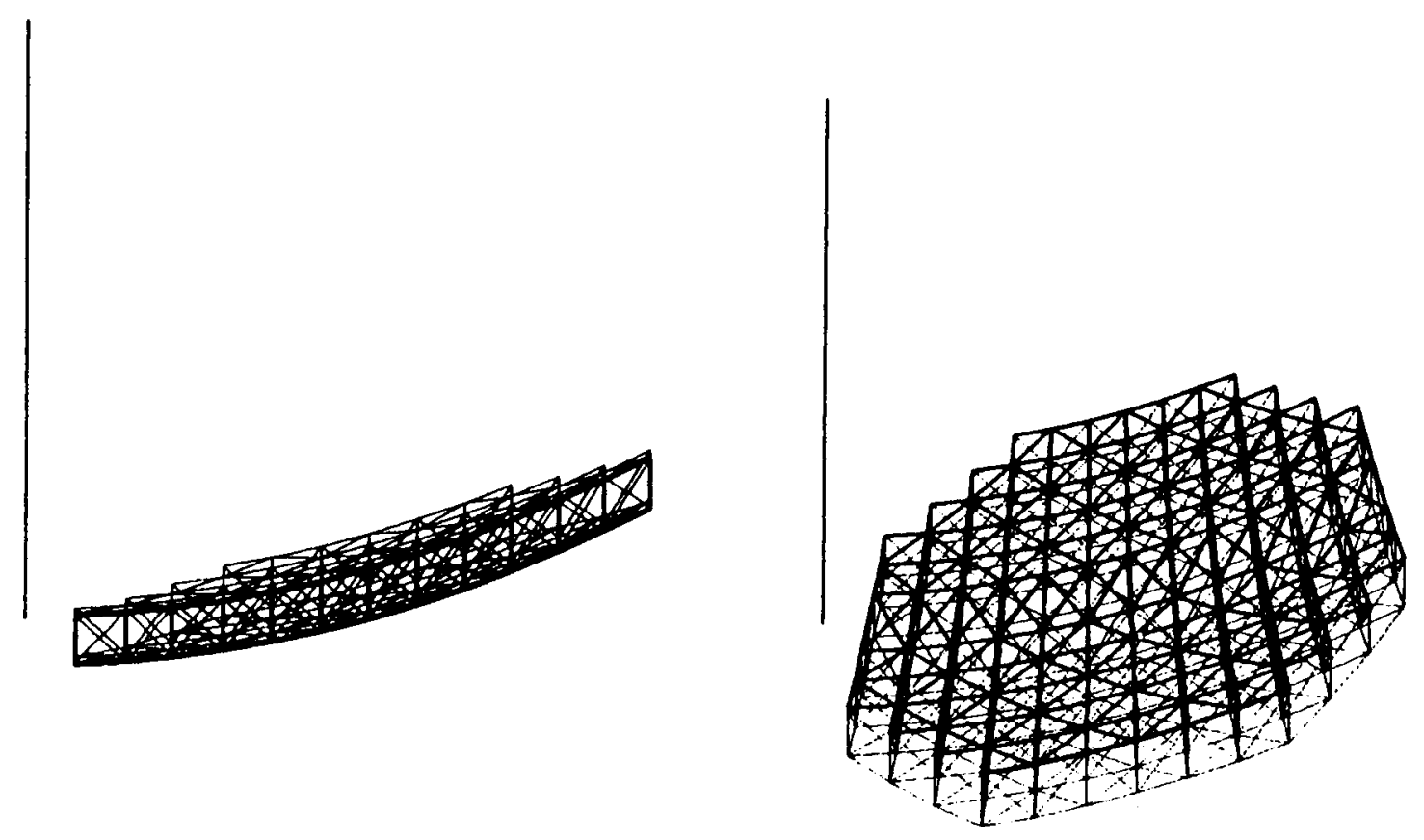

Elevation View

Perspective View

Figure 15. Views of Pactruss for offset paraboloid. 


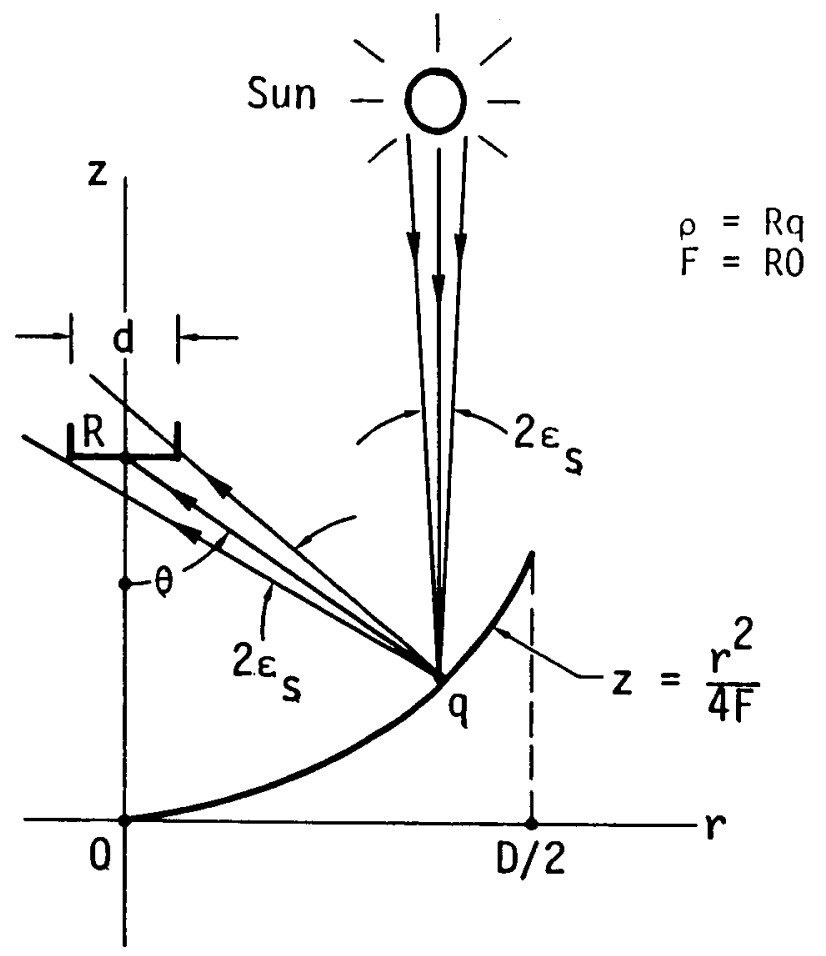

Figure 16. Geometry of a continuous paraboloid reflector.

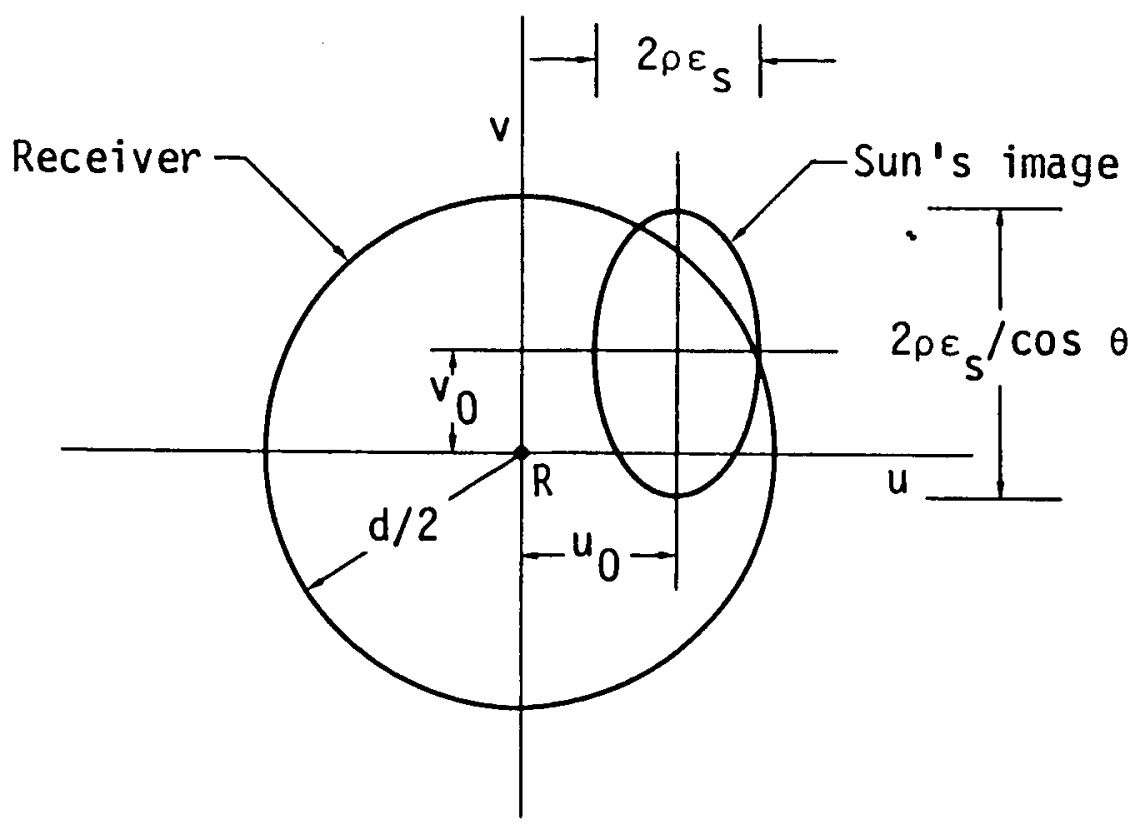

Figure 17. Intersection of the sun's image and the receiver disk in the receiver plane. 


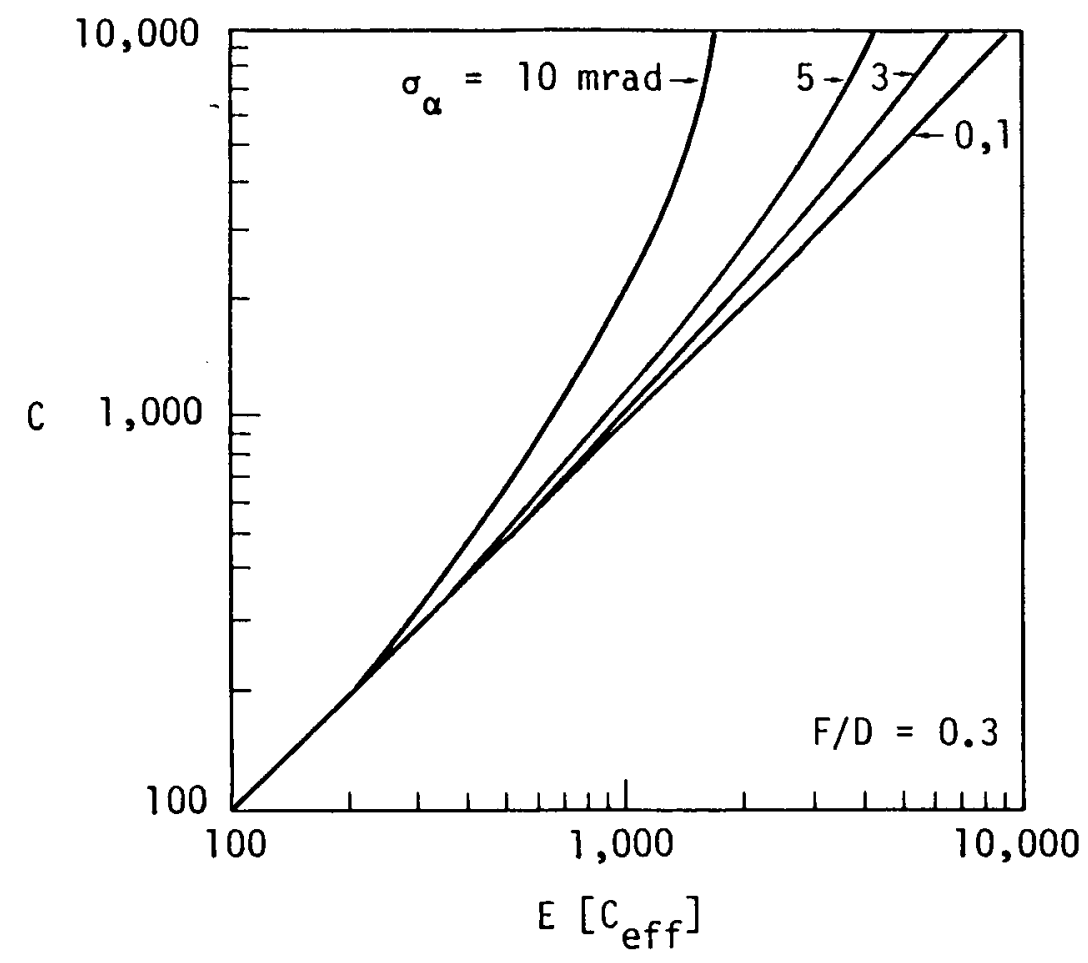

86-1101

Figure 18. Mean effective concentration ratio vs. geometric concentration ratio $C$ with $\mathrm{rms}$ surface slope error $\sigma_{\alpha}(F / D=0.3)$.

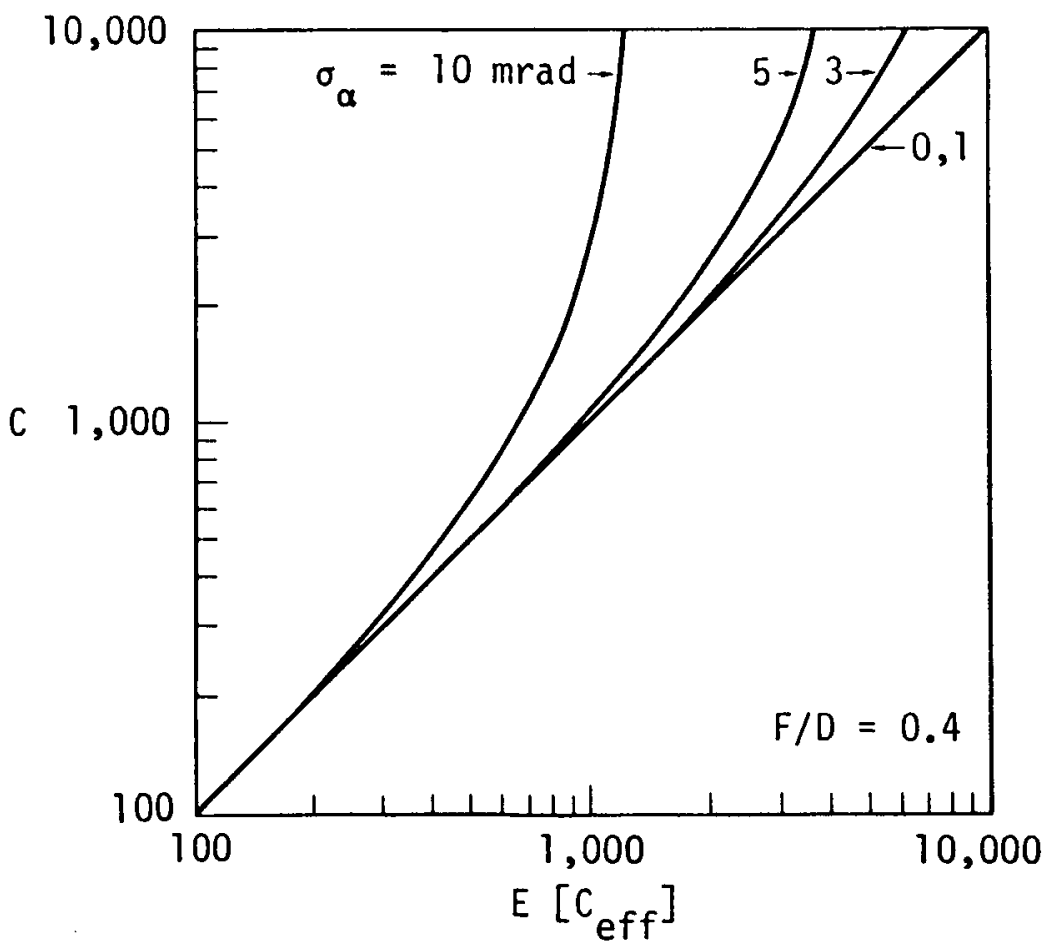

$86-1102$

Figure 19. Mean effective concentration ratio vs. geometric concentration ratio $C$ with rms surface slope error $\sigma_{\alpha}(F / D=0.4)$. 


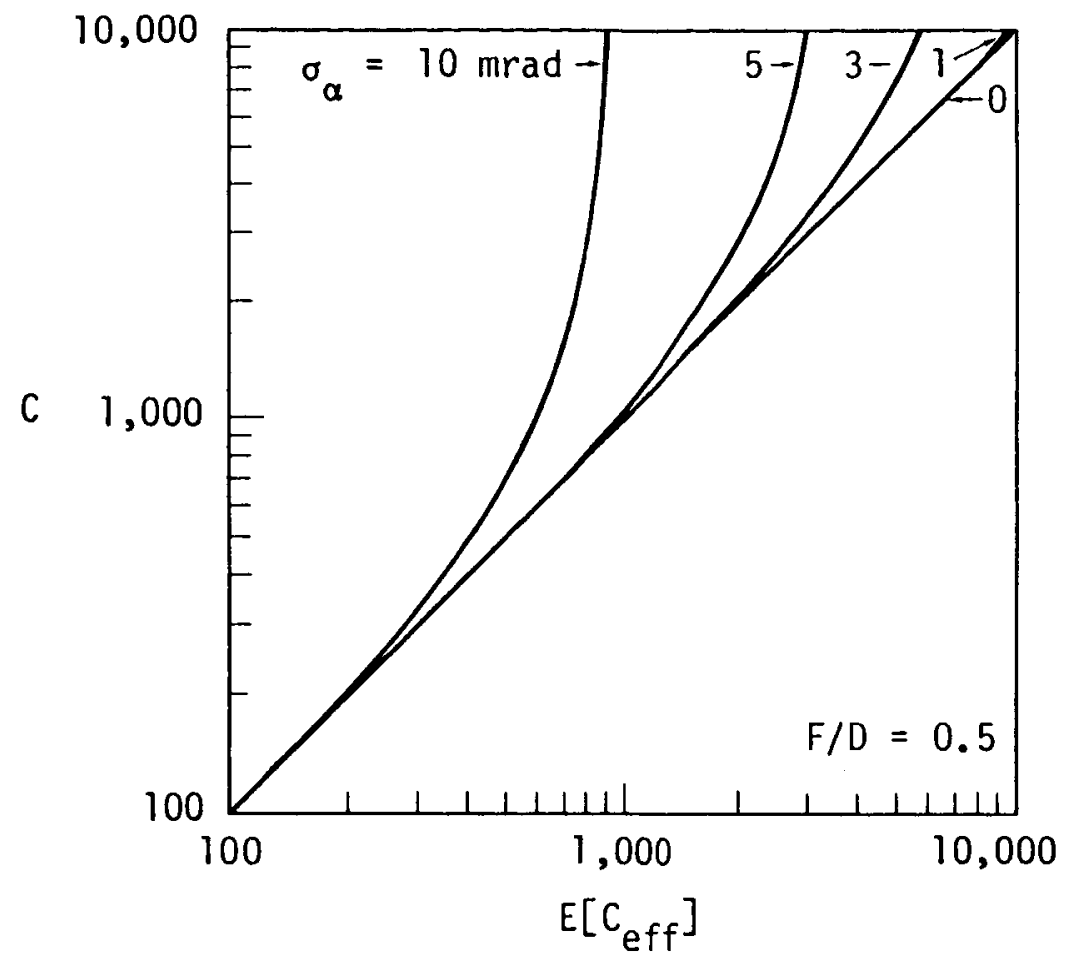

86-1103

Figure 20. Mean effective concentration ratio vs. geometric concentration ratio $C$ with rms surface slope error $\sigma_{\alpha}(F / D=0.5)$.

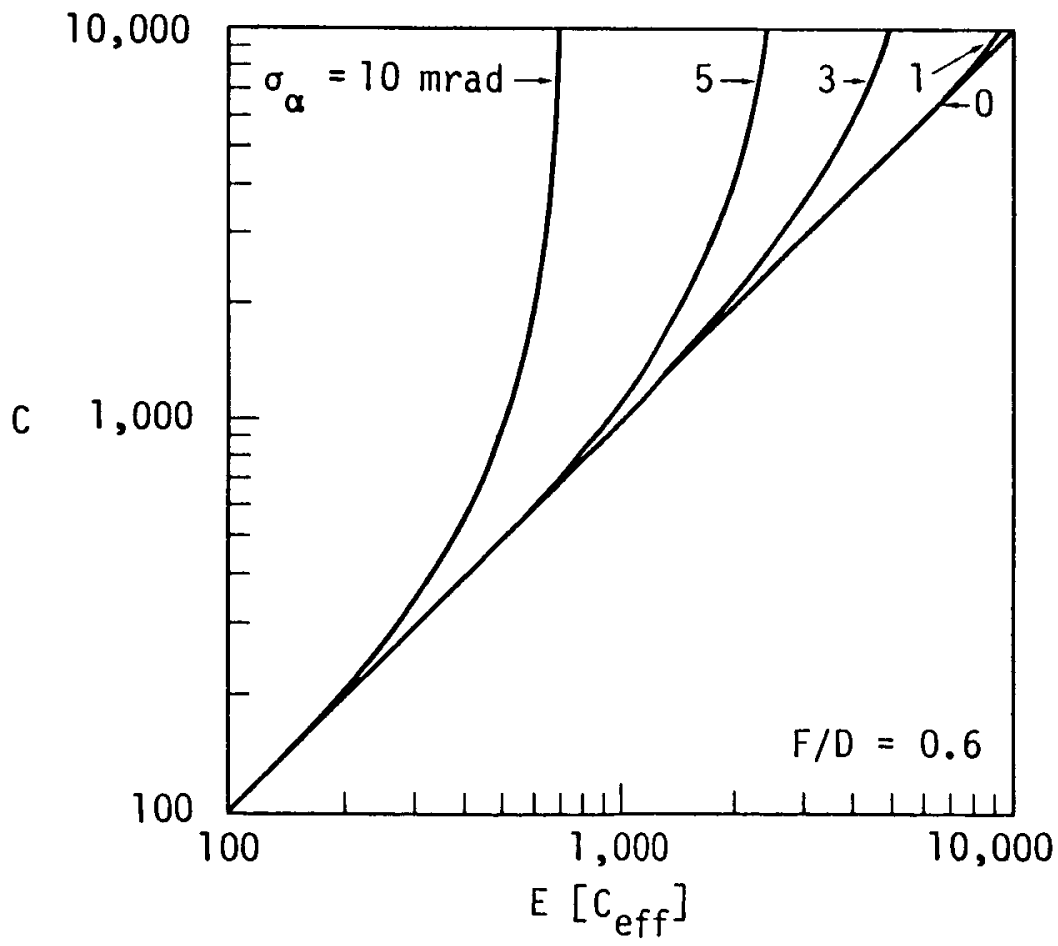

86-1104

Figure 21. Mean effective concentration ratio vs. geometric concentration ratio $C$ with rms surface slope error $\sigma_{\alpha}(F / D=0.6)$. 


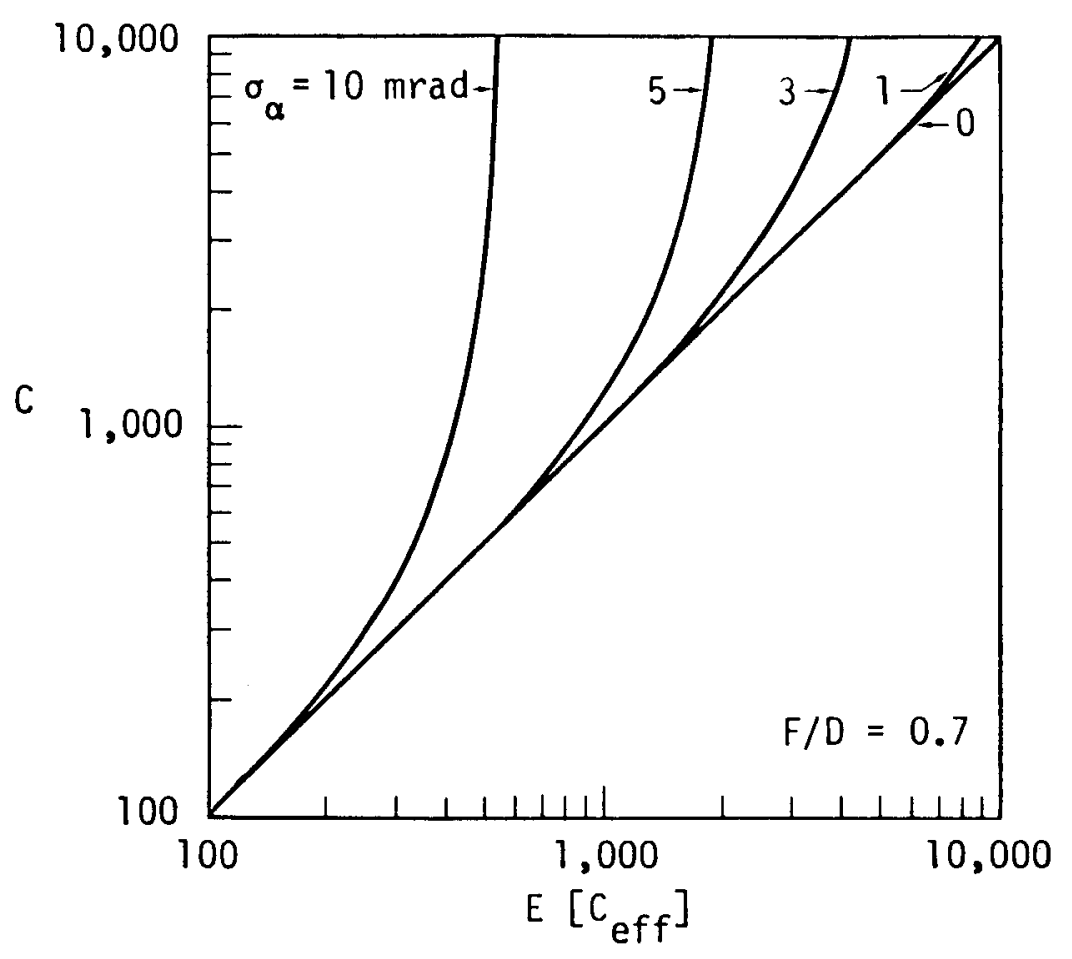

86-1105

Figure 22. Mean effective concentration ratio vs. geometric concentration ratio $\mathrm{C}$ with rms surface slope error $\sigma_{\alpha}(F / D=0.7)$.

86-1106

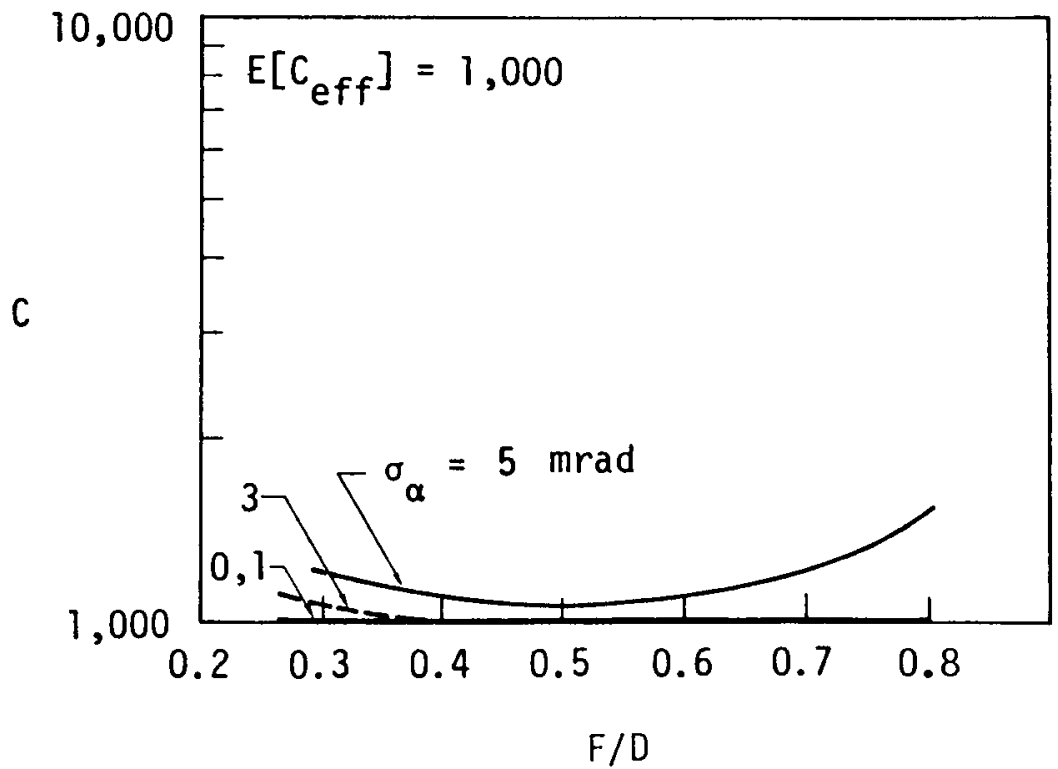

Figure 23. Effect of $F / D$ on required geometric concentration ratio $C$ with rms surface slope error $\sigma_{\alpha}$ (E $\left.\left[C_{\text {eff }}\right]=1,000\right)$. 


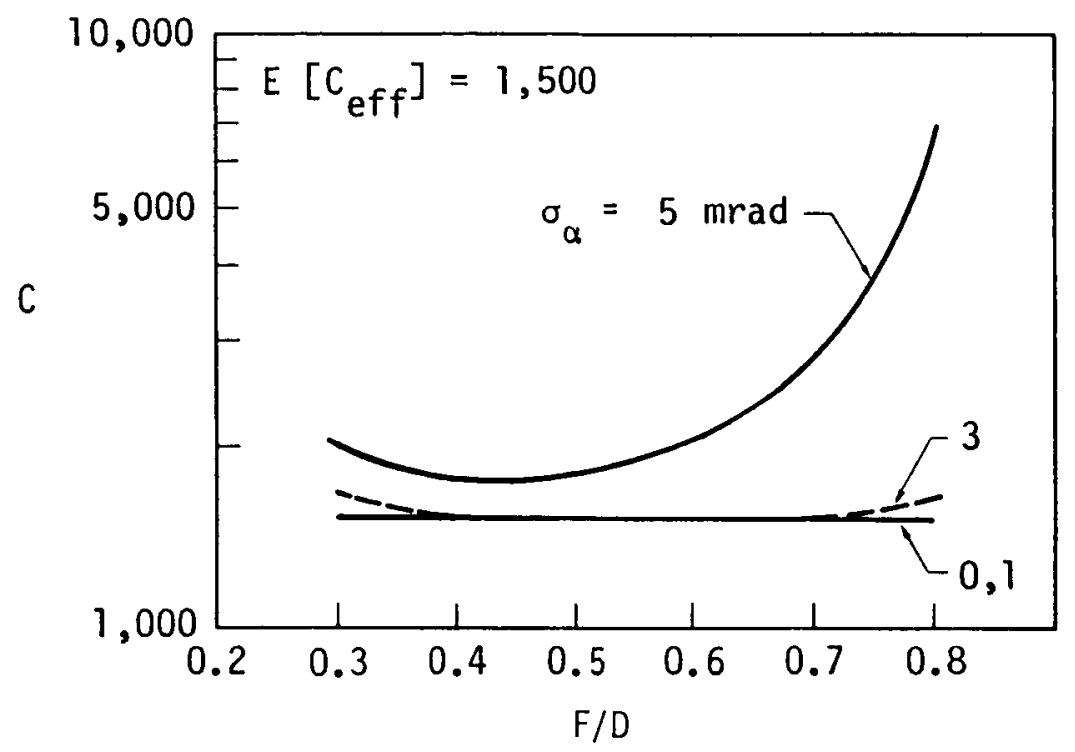

Figure 24. Effect of $F / D$ on required geometric concentration ratio $C$ with rms surface slope error $\sigma_{\alpha}$ $\left(E\left[C_{\text {eff }}\right]=1,500\right)$.

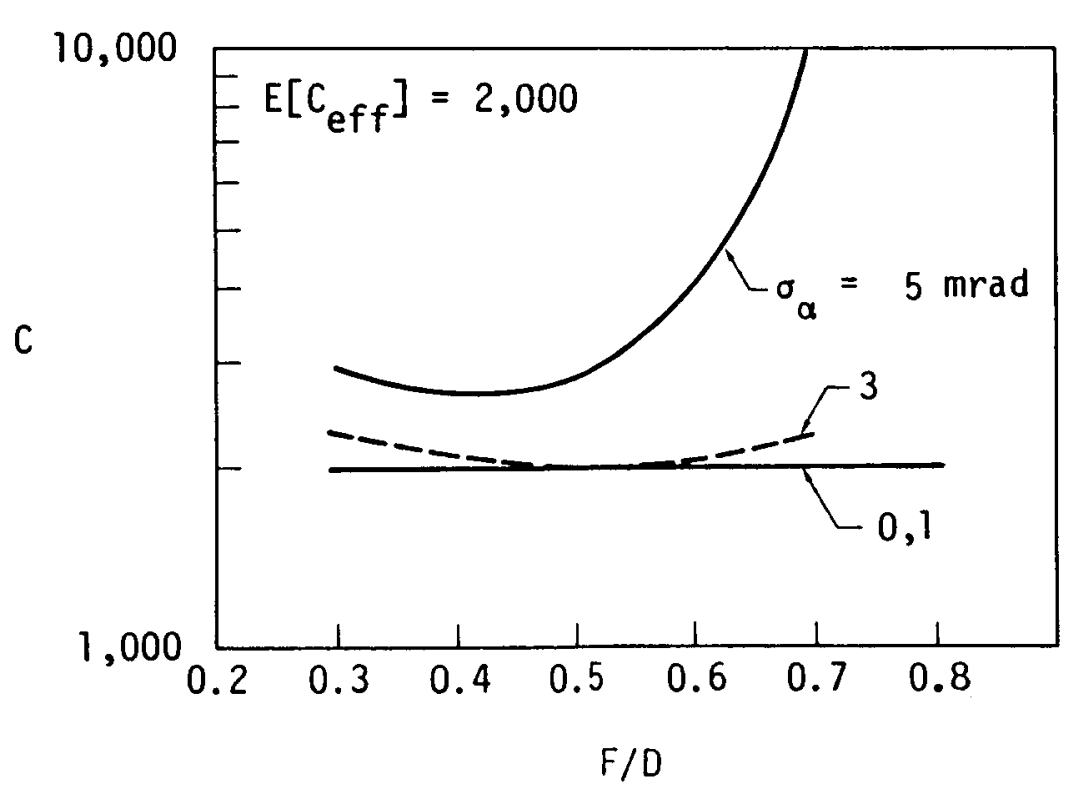

$86-1108$

Figure 25. Effect of $F / D$ on required geometric concentration ratio $C$ with rins surface slope error $\sigma_{a}$ $\left(E\left[C_{\text {eff }}\right]=2,000\right)$. 


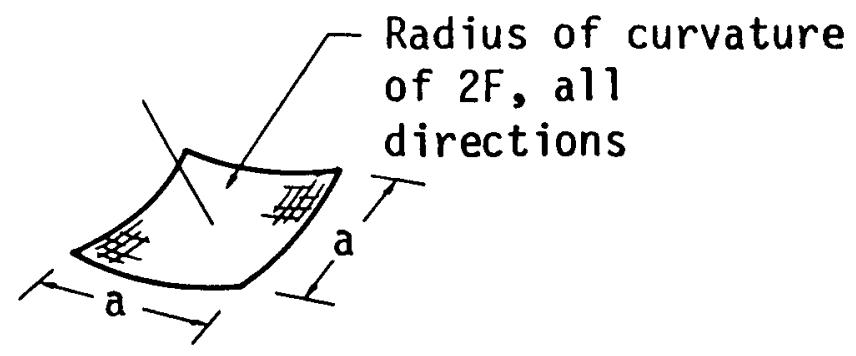

Figure 26. Nominally square shallow spherical panel with side length "a".

$86-1112$

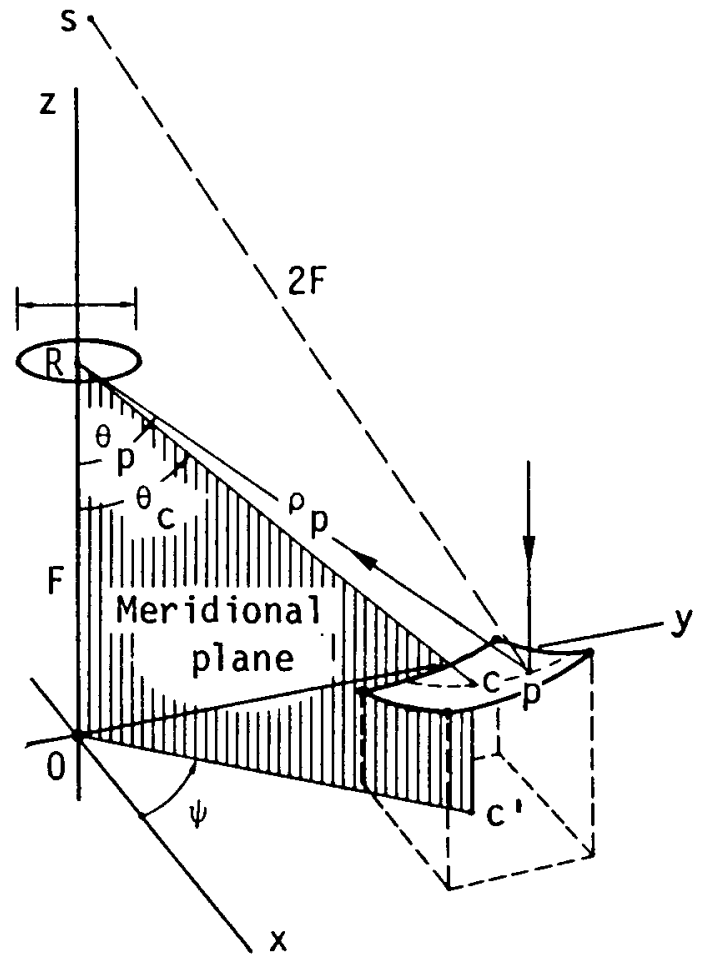

Figure 27. Location and orientation geometry of square spherical panels. 


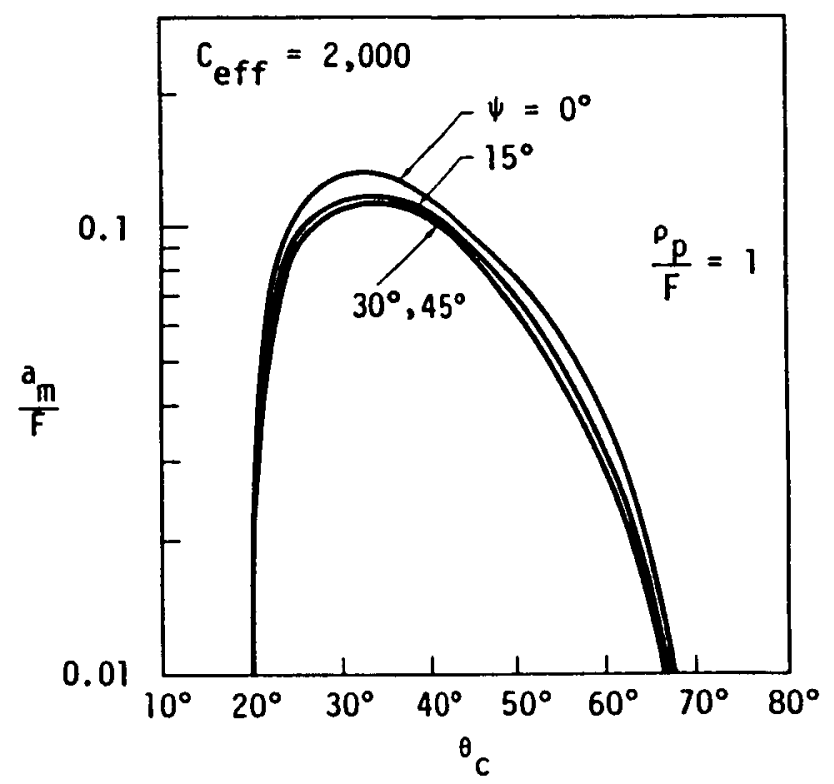

Figure 28. Effect of panel orientation angle $\psi$ on maximum panel size $a_{m} / F$ vs. panel location angle $\theta_{c}$.

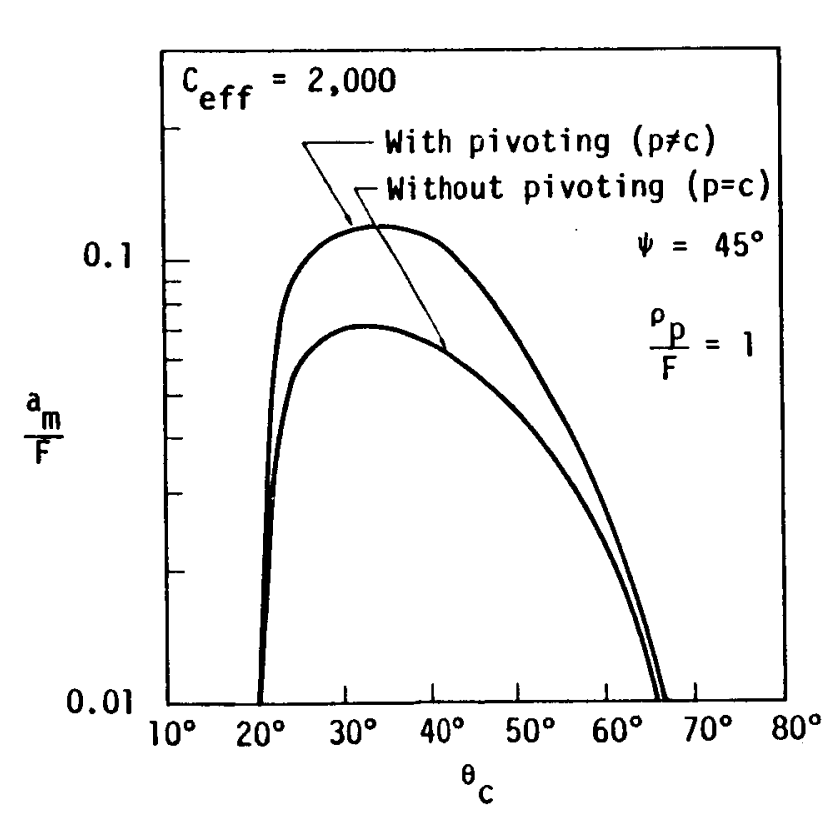

Figure 29. Effects of pivoting the panel so that $p$ and $c$ are not coincident, on the maximum allowable panel size for the case where $C_{\text {eff }}=2,000$, $\rho_{p}=F$, and $\psi=45$ degrees. 


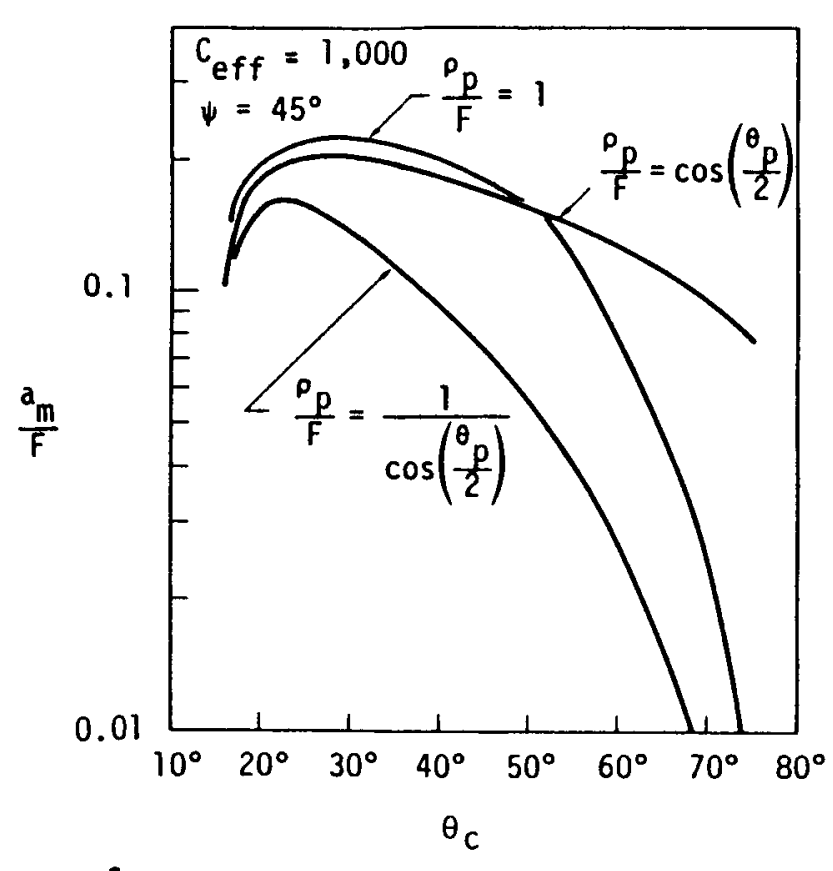

$86-1110$

Figure 30. Maximum panel size $a_{m} / F$ vs. panel location angle $\theta_{c}$ with different panel distances $\rho_{p} / F$.

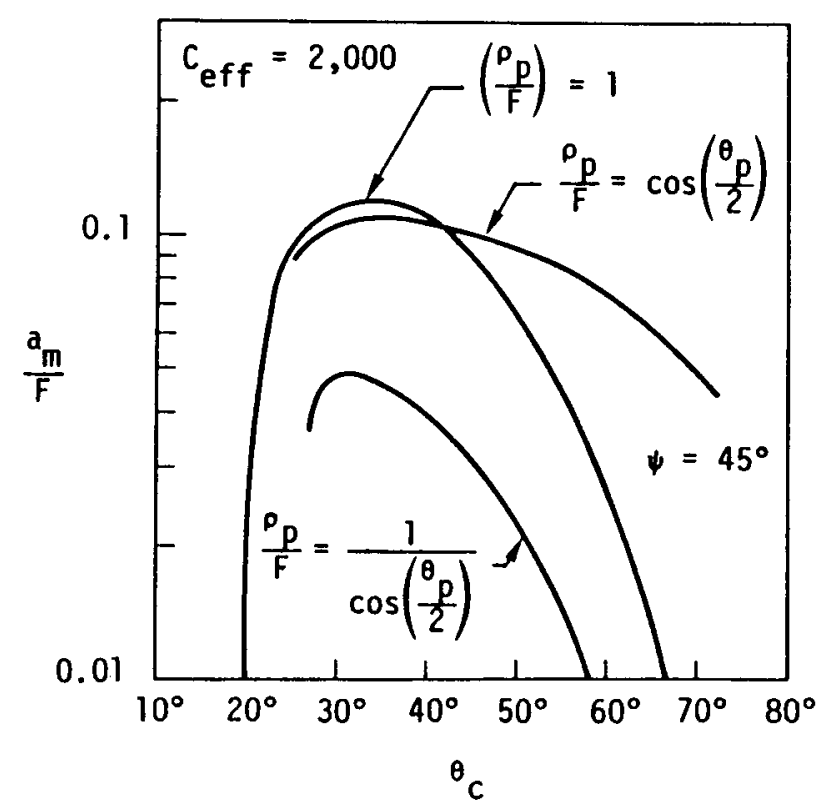

Figure 31. Maximum panel size $a_{m} / F$ vs. panel location angle $\theta_{c}$ with different panel distances $\rho_{p} / F$. 


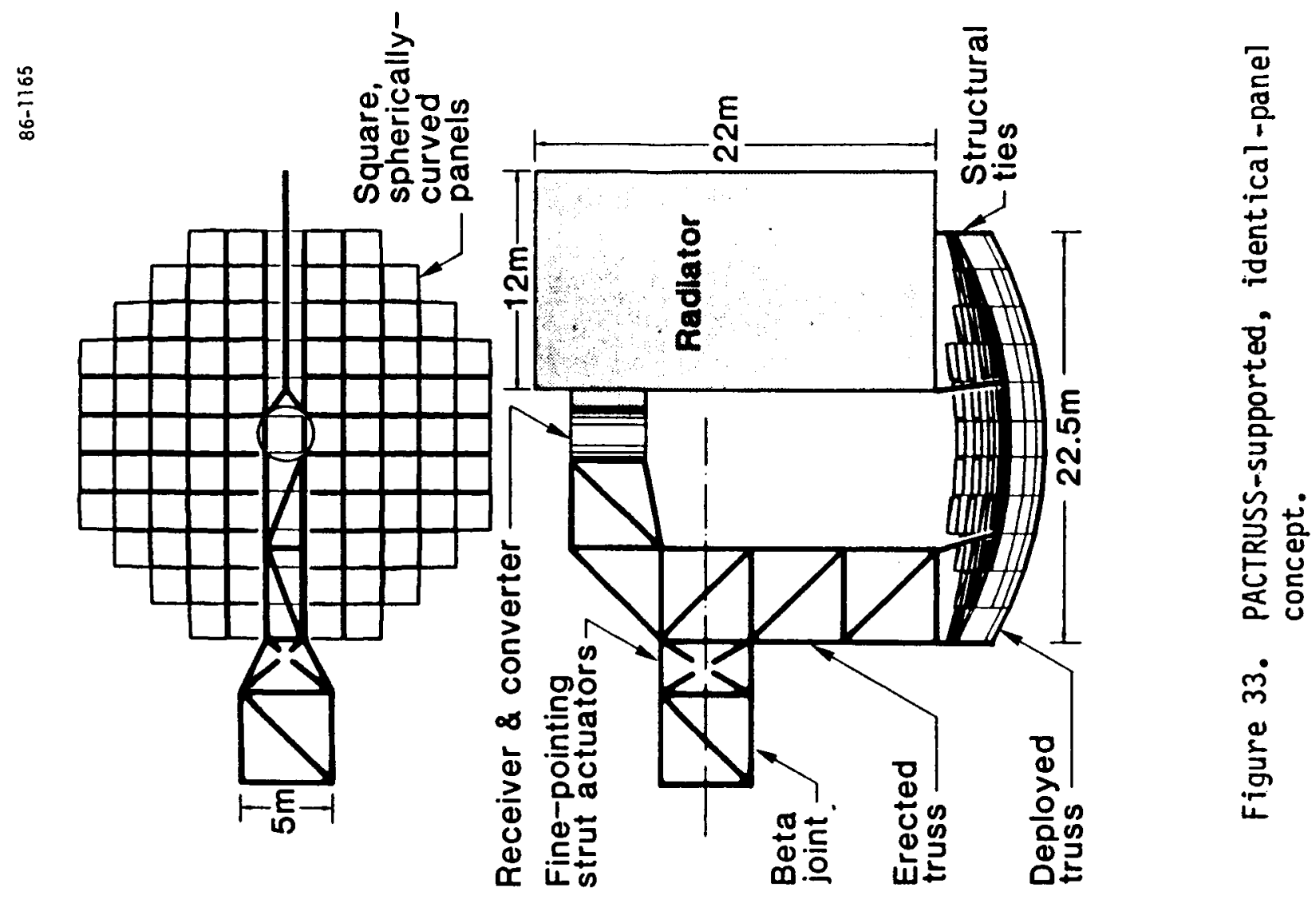

点

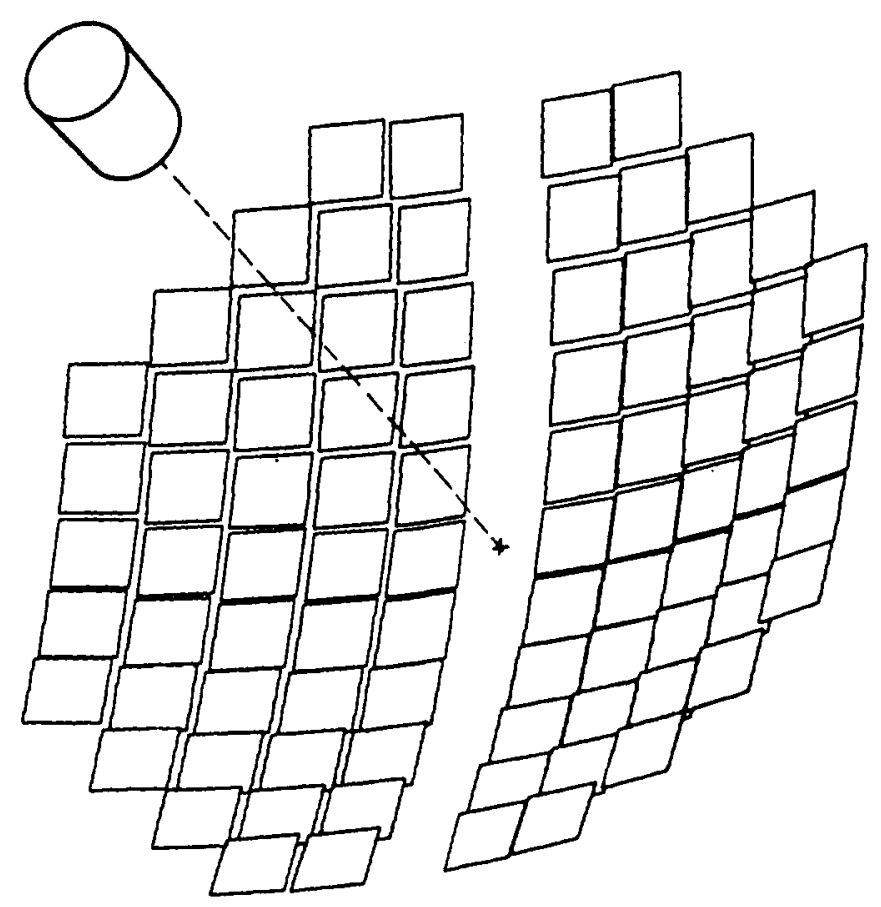

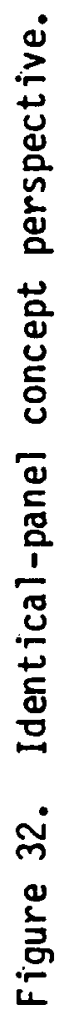


APPENDIX 


\section{APPENDIX}

/* SOLARPAC.C - Program for generating the design geometry of a solar concentrator made up of identical square panels supported by a Pactruss structure.

$$
\text { jmh } 1 / 3 / 86 \quad * /
$$

\#include "math.h"

struct geom \{

double focal;

double size;

double tilt;

\} ;

double corner_coord[4] [3];

main()

\{

int $i, j, n, m, d t a \_f i l e, f l a g, N$, Nnodes, Ncorners, Nmembers, Ntotal;

int Ntypes, chan;

char chr;

double a, l, H, h, F, templ, temp2, r, theta, phi , xc, yc, zc, xl, yl, zl;

double *nodes, *corners, *ptemp, *ptr, *pcorn;

struct geom *tile_ptr;

static double accuracy $=1.0 \mathrm{E}-12$;
$\mathbf{a}=1.95$
$1=2.1$;
$\mathrm{H}=1$;
$\mathrm{h}=0$;
$\mathrm{F}=21$.
$N=5$;

/* Panel size

/* Bay length

/* Truss height

/* Vertical panel offset */

/* Focal length

*/

/* Radial number of panels */

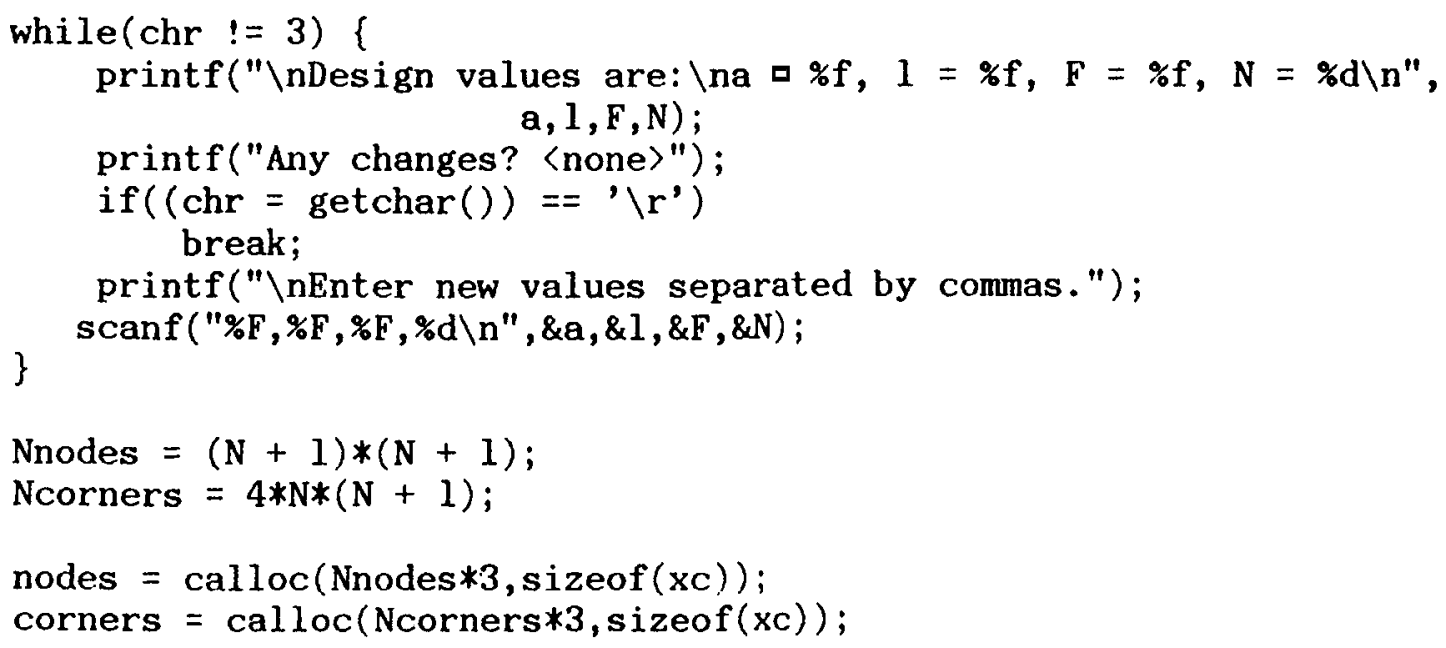




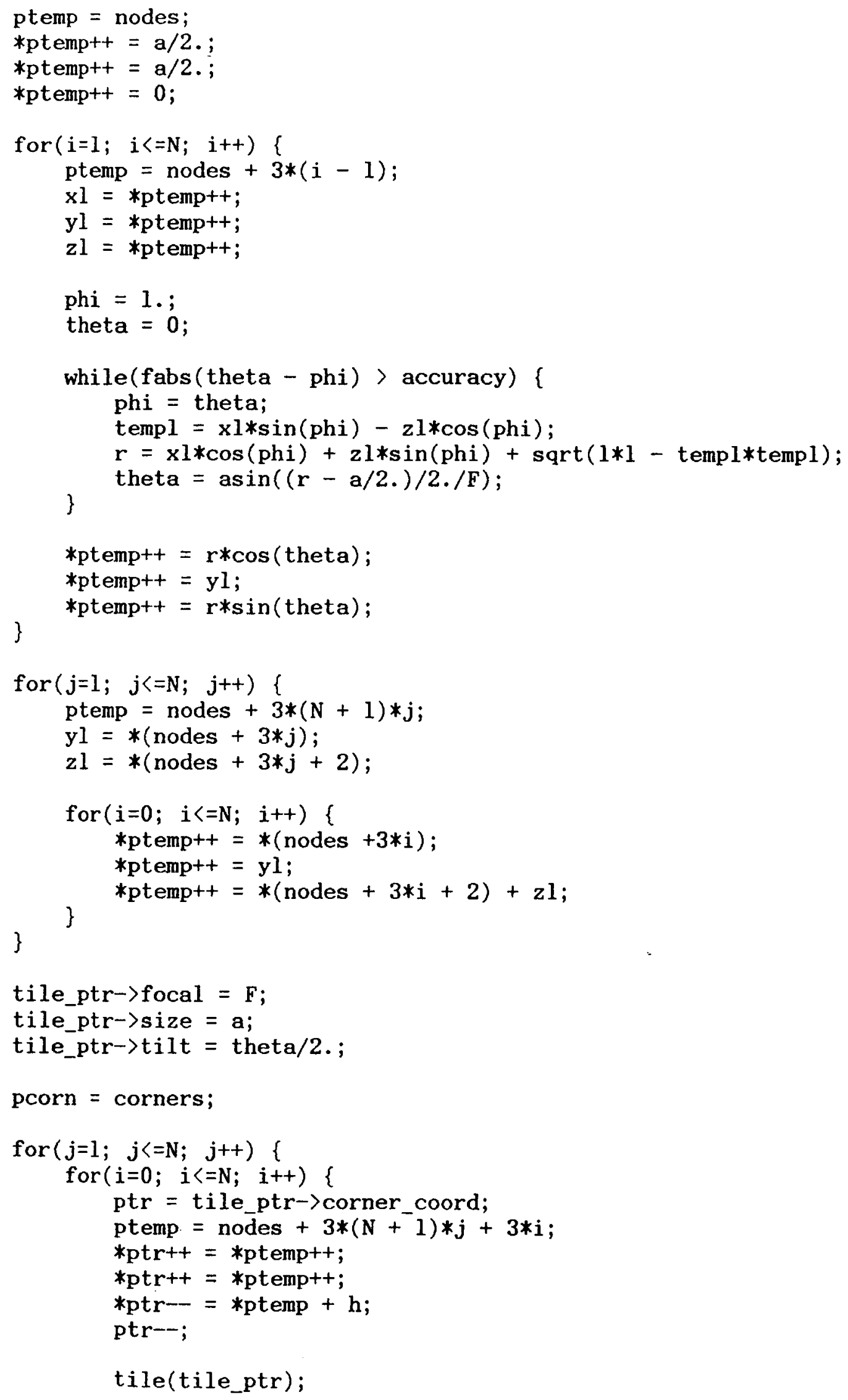




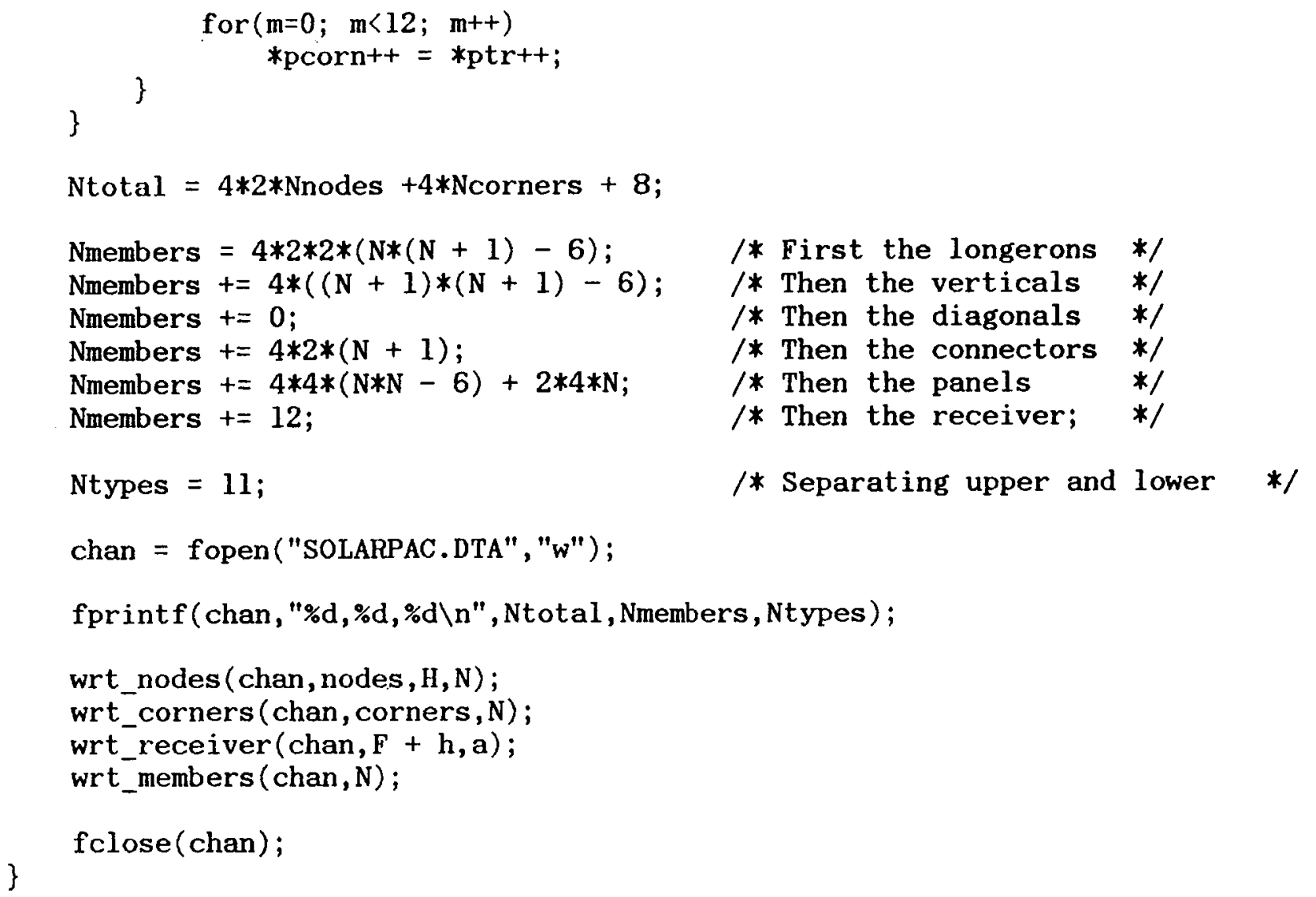

/* TILE.C - Determines the locations of the corners of a square reflector panel after rotation from the horizontal to the desired orientation.

jmh 12/29/85 */

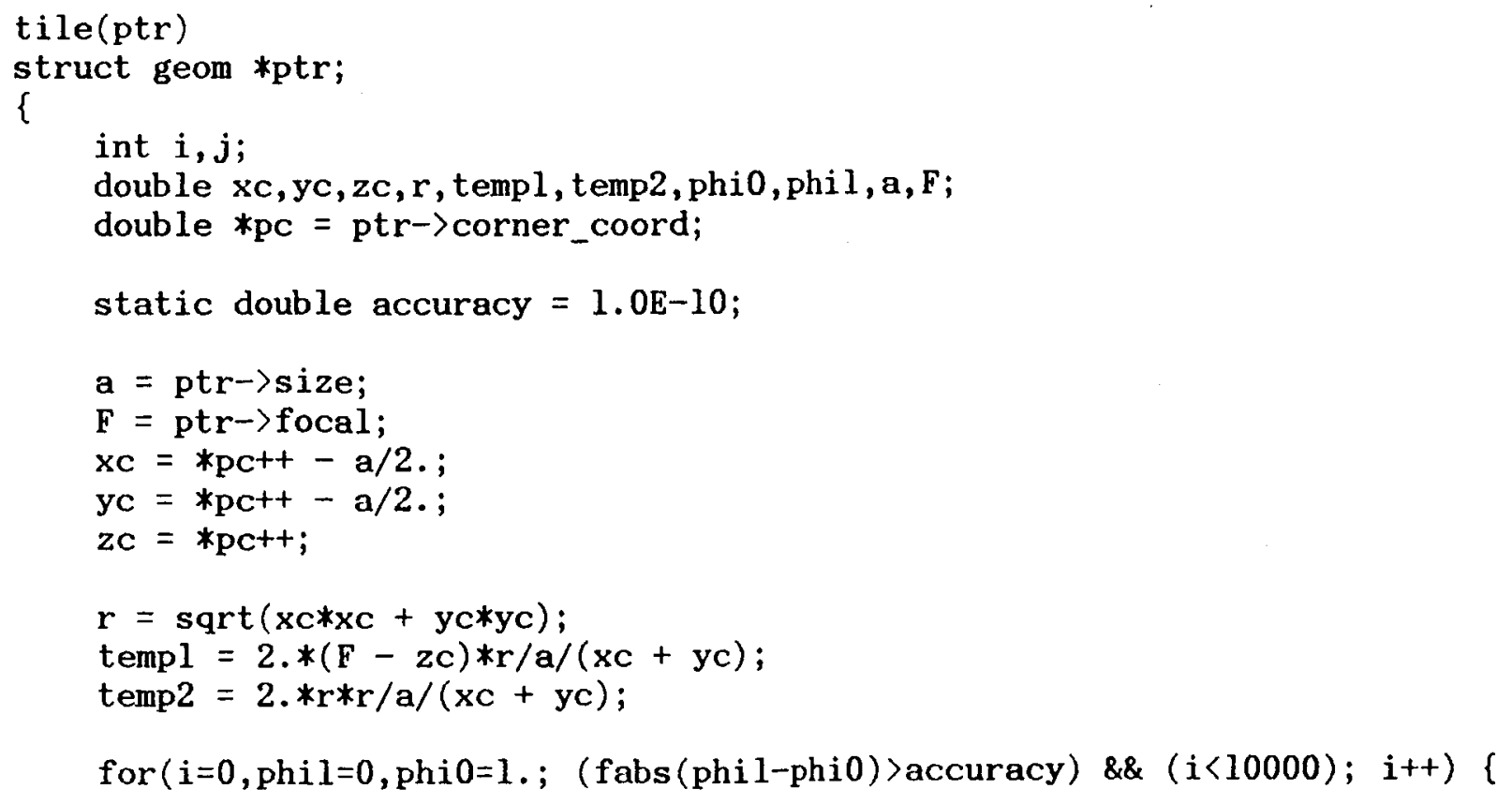




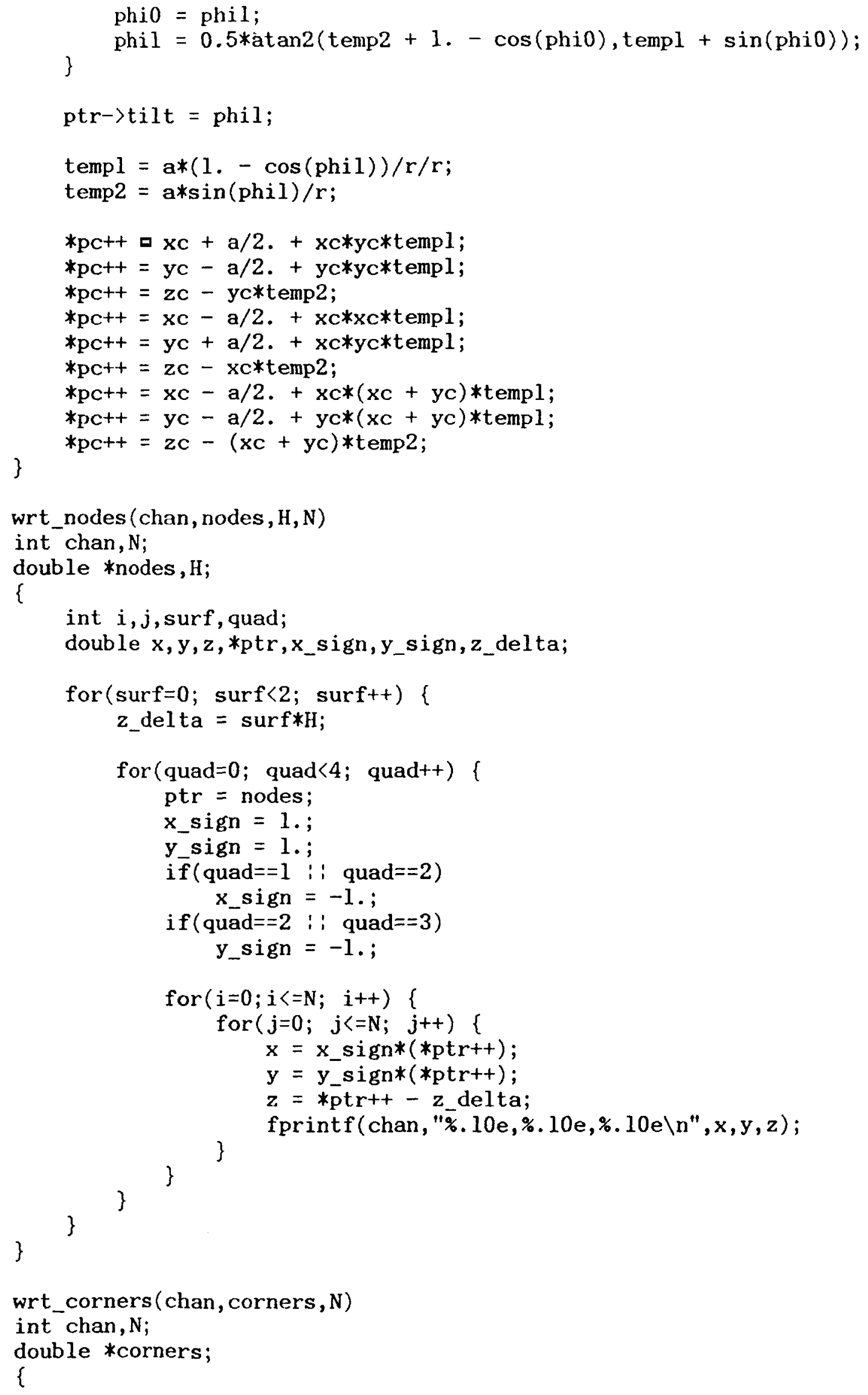




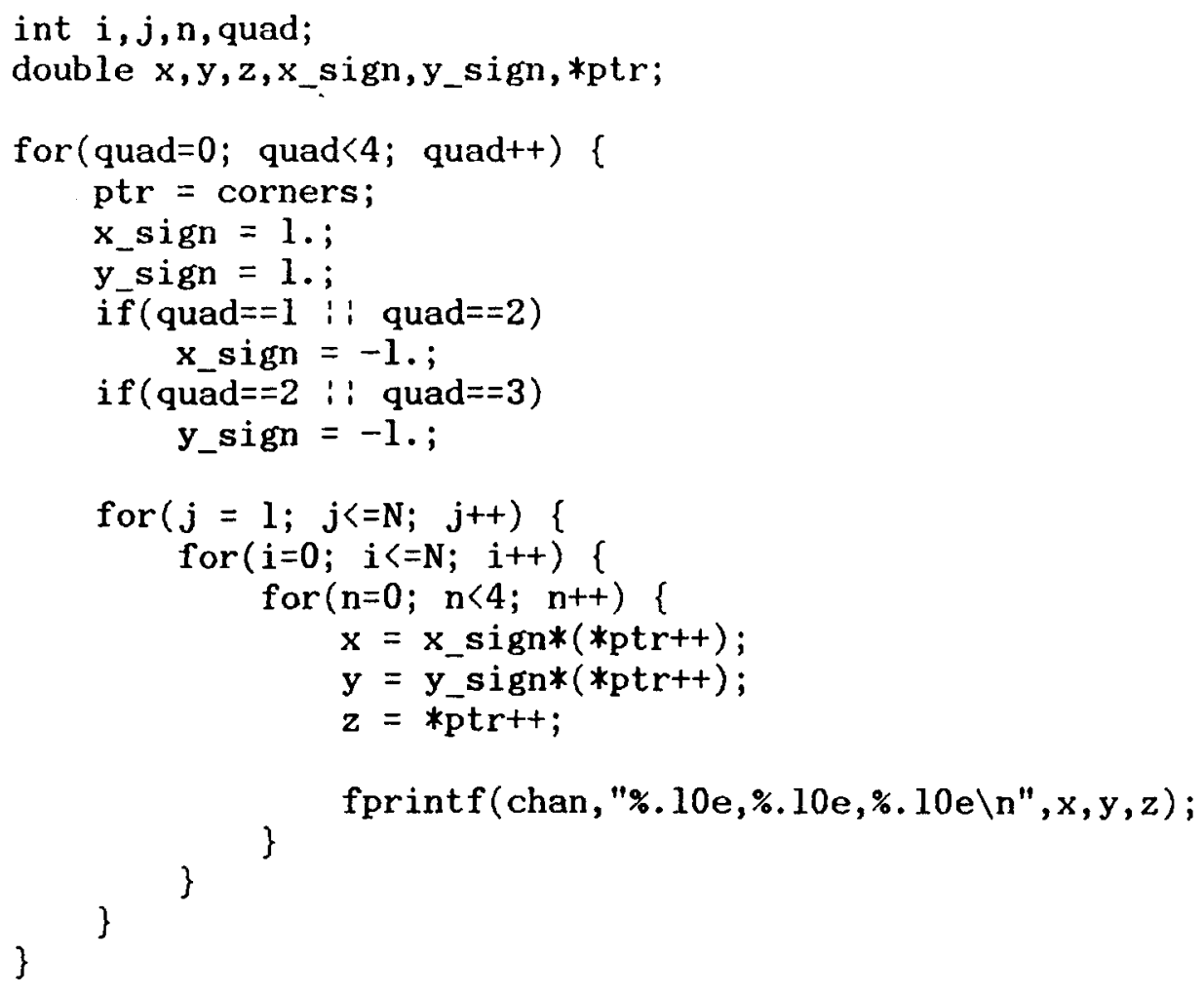




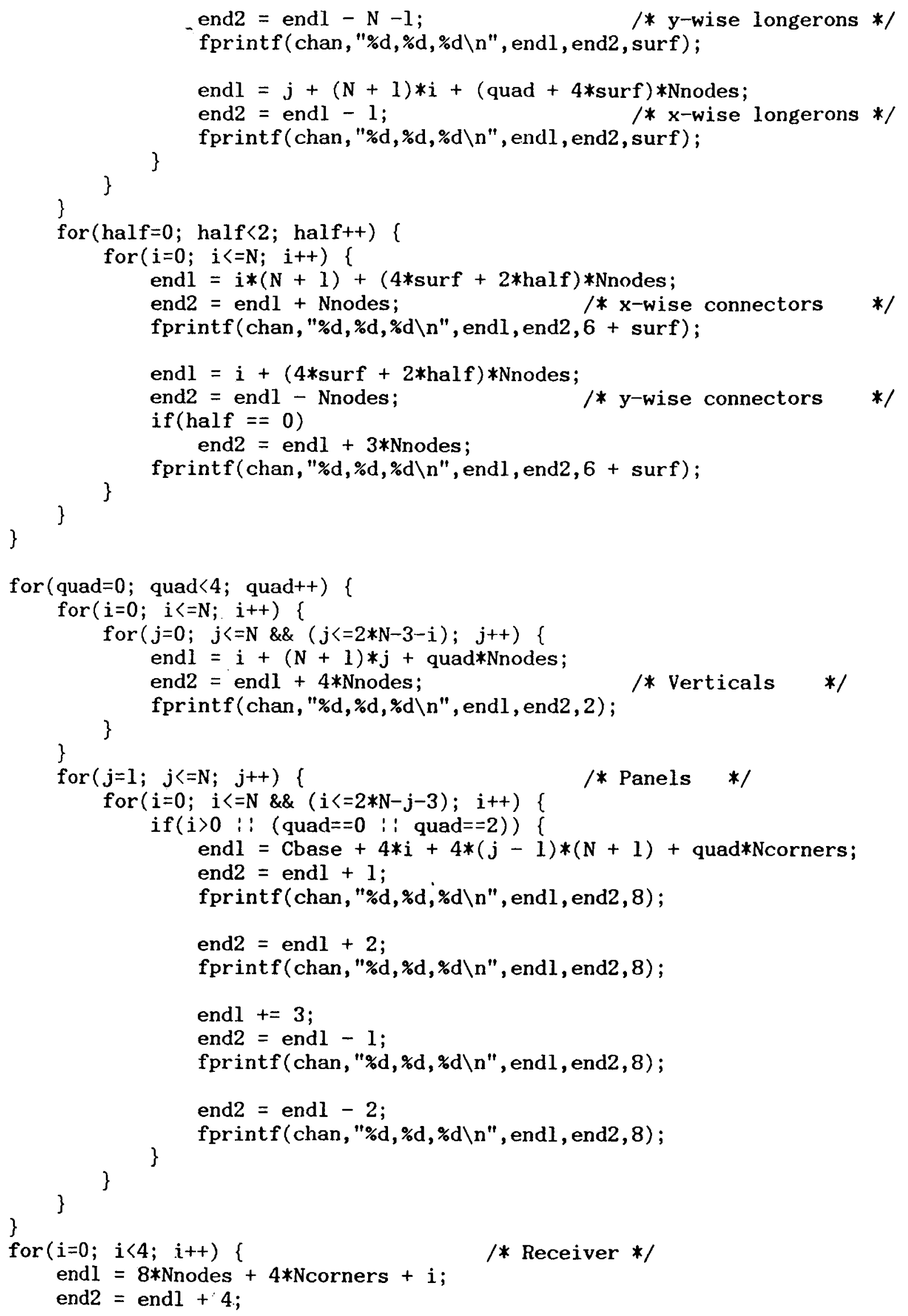


\}

fprintf (chan, "\%d, \%d,\%d\n", endl, end2, 9) ;

for $(i=0 ; i<2 ; i++)($

endl $=8 *$ Nnodes $+4 *$ Ncorners $+4 * i$;

end2 $=$ end $1+1$;

fprintf (chan, "\%d,\%d,\%d\n", endl, end2, 9+i);

end2 $=$ endl +2 ;

fprintf (chan, "\%d, \%d,\%d \n", endl, end2, 9+i) ;

endl $t=3$;

end2 $=$ end $1-1$;

fprintf(chan, "\%d, \%d, \%d \n", end1, end2, 9+i);

end2 = endl -2 ;

fprintf (chan, "\%d, \%d,\%d\n", endl, end2, 9+i);

\}

$$
\text { \} }
$$$$
\text { (chan, \%d, \%d, \%d \n", endl, end2, 9+i); }
$$ 
Standard Bibliographic Page

\begin{tabular}{|c|c|}
\hline 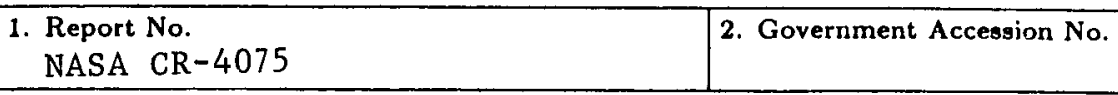 & 3. Recipient's Catalog No. \\
\hline \multirow{2}{*}{$\begin{array}{l}\text { 4. Title and Subtitle } \\
\text { Structural Concepts for Large Solar } \\
\text { Concentrators }\end{array}$} & $\begin{array}{l}\text { 5. Report Date } \\
\text { June } 1987\end{array}$ \\
\hline & $\begin{array}{l}\text { 6. Performing Organization Code } \\
\text { AAC-TN-1146, Rev. A }\end{array}$ \\
\hline $\begin{array}{l}\text { 7. Author(s) } \\
\text { John M. Hedgepeth and Richard K. Miller }\end{array}$ & 8. Performing Organization Report No. \\
\hline \multirow{2}{*}{$\begin{array}{l}\text { 9. Performing Organization Name and Address } \\
\text { Astro Aerospace Corporation } \\
6384 \text { Via Real } \\
\text { Carpinteria, CA. } 93013-2993\end{array}$} & 10. Work Unit No. \\
\hline & $\begin{array}{l}\text { 11. Contract or Grant No. } \\
\text { NAS } 1-17536 \text {, Task } 3\end{array}$ \\
\hline \multirow{2}{*}{$\begin{array}{l}\text { 12. Sponsoring Agency Name and Address } \\
\text { National Aeronautics and Space Administration } \\
\text { Washington, DC } 20546\end{array}$} & $\begin{array}{l}\text { 13. Type of Report and Period Covered } \\
\text { Contractor Report }\end{array}$ \\
\hline & $\begin{array}{l}\text { 14. Sponsoring Agency Code } \\
481-32-23-01\end{array}$ \\
\hline
\end{tabular}

15. Supplementary Notes

Langley Technical Monitor: W. B. Fichter

Final Report

16. Abstraclast concepts originating in the 1960 s are discussed. These include stiff sandwich panels and aluminum dishes as well as inflated and umbrella-type membrane configurations. The Sunflower concentrator, developed in the early 1970s, is described as a salient example of a high-efficiency concentrator. The newly emphasized needs for solar dynamic power on the Space Station and for large, lightweight thermal sources are outlined.

Existing concepts for high efficiency reflector surfaces are examined with attention to accuracy needs for concentration ratios of 1,000 to 3,000 . Concepts using stiff reflector panels are deemed most likely to exhibit the long-term consistent accuracy necessary for low-orbit operation, particularly for the higher concentration ratios. Quantitative results are shown of the effects of surface errors for various concentration and focal-length-diameter ratios.

The costs of providing high efficiency are discussed. Principal sources of high cost include the need for variously dished panels for paraboloidal reflectors and the the expense of ground testing and adjustment. A new configuration is presented that addresses both of these problems. It consists of a deployable Pactruss backup structure with identical panels installed on the deployed structure after deployment in space. Analytical results show that with reasonable pointing errors, this new concept is capable of concentration ratios greater than 2,000 .

\begin{tabular}{|c|c|c|c|}
\hline $\begin{array}{l}\text { 17. Key Words (Suggested by Authors(s)) } \\
\text { Space power } \\
\text { Space structures } \\
\text { Deployable structures } \\
\text { Solar concentrators } \\
\text { Segmented reflectors } \\
\text { Structural concepts }\end{array}$ & $\begin{array}{l}\text { 18. Distribution State } \\
\text { Unclassifie }\end{array}$ & $\begin{array}{l}\text { - Unlimite } \\
\text { Subject Ca }\end{array}$ & $\begin{array}{l}\text { d } \\
\text { tegory } 18\end{array}$ \\
\hline $\begin{array}{l}\text { 19. Security Classif.(of this report) } \\
\text { Unclass ified }\end{array}$ & $\begin{array}{l}\text { 20. Security Classif.(of this page) } \\
\text { Unclassified }\end{array}$ & $\begin{array}{c}\text { 21. No. of Pages } \\
63\end{array}$ & $\begin{array}{r}\text { 22. Price } \\
\text { A04 }\end{array}$ \\
\hline
\end{tabular}

